\title{
Compression and dilation of the membrane-cortex layer generates rapid changes in cell shape
}

\author{
Maryna Kapustina, ${ }^{1}$ Timothy C. Elston, ${ }^{2,3}$ and Ken Jacobson ${ }^{1,3}$ \\ 'Department of Cell Biology and Physiology, ${ }^{2}$ Department of Pharmacology, and ${ }^{3}$ Lineberger Comprehensive Cancer Center, University of North Carolina School of \\ Medicine, Chapel Hill, NC 27599
}

\section{Introduction}

Mounting the appropriate response to an environmental challenge often involves large-scale changes in cell morphology (Janmey and McCulloch, 2007; Kasza et al., 2007; Hoffman et al., 2011; Kasza and Zallen, 2011). For example, environmental cues such as hormones or growth factors can lead to cell differentiation, proliferation, or migration. Nearly all aspects of cell movement are tightly regulated by a signaling network that includes phosphoinositides and the Rho family of small GTPases (Servant et al., 1999; Logan and Mandato, 2006; Machacek et al., 2009; Brill et al., 2011; Provenzano and Keely, 2011). These molecules play central roles in regulating the actin cortex, the filamentous meshwork that lies adjacent to the cell membrane and generates the contractile forces required for changes in cell morphology (Pesen and Hoh, 2005; Hawkins et al., 2011; Rangamani et al., 2011; Sedzinski et al., 2011).

Cells in 3D tissue often exhibit rounder morphologies and migrate via substantially different mechanisms than those used in migration on 2D substrates (Lorentzen et al., 2011; Rottner and Stradal, 2011; Tsujioka, 2011). However, studies of cell shape transformations within extracellular matrix tissue present substantial challenges owing to the complexity of the environment

Correspondence to Maryna Kapustina: mkapust@med.unc.edu

Abbreviations used in this paper: DIC, differential interference contrast; PM, plasma membrane; PMT-mRFP, PM target monomeric red fluorescent protein; TEM, transmission electron microscopy.

membrane-cortex traveling wave led to amoeboid-like cell migration. The compression-dilation hypothesis offers a mechanism for large-scale cell shape transformations that is complementary to blebbing, where the plasma membrane detaches from the actin cortex and is initially unsupported when the bleb extends as a result of cytosolic pressure. Our findings provide insight into the mechanisms that drive the rapid morphological changes that occur in many physiological contexts, such as amoeboid migration and cytokinesis. and the difficulty in obtaining images that are of quality comparable to those obtained for 2D migration. The periodic morphological protrusions (oscillations) exhibited by many rounded cells may represent a simpler model system to study amoeboidlike cell protrusions that are tractable from both experimental and theoretical points of view (Pletjushkina et al., 2001; Paluch et al., 2005; Salbreux et al., 2007; Kapustina et al., 2008; Costigliola et al., 2010).

In this study, we demonstrated that compression (folding) and subsequent dilation (unfolding) of the plasma membrane (PM)-cortex layer underlies the periodic protrusive phenotype (we use this term because oscillating cells exhibit rounded protrusions at a defined frequency) and may provide a general mechanism for rapid transformations in cell shape. We found that fluorescent signals from the PM and the F-actin cortex are highly correlated in all stages of protrusion and they are both inversely correlated with protrusion size. We discovered that oscillations can be initiated as a result of spread cells transitioning to a rounded state when cells must store excess surface area in folds. Membrane-cortex folding in the periodic protrusive phenotype was confirmed by electron microscopy. We found

(c) 2013 Kapustina et al. This article is distributed under the terms of an AttributionNoncommercial-Share Alike-No Mirror Sites license for the first six months after the publication date (see http://www.rupress.org/terms). After six months it is available under a Creative Commons License (Attribution-Noncommercial-Share Alike 3.0 Unported license, as described at http://creativecommons.org/licenses/by-nc-sa/3.0/). 
Figure 1. Cytoskeletal dynamics during periodic protrusions. (A) DIC and Lifeact-GFP wide-field fluorescent images of an oscillating cell. (B) Cytoskeletal dynamics during one period of oscillation (Video 1). Merged fluorescence images of highly polarized F-actin (green) and myosin II (red) throughout the oscillatory cycle. (C) Laser scanning confocal images and corresponding plots of the fluorescent signal from F-actin (Lifeact-GFP) of one representative oscillating cell $(n=35)$ for two time points. (left) Green rectangles $(8.5 \times 1.5 \mu \mathrm{m})$ show the positions where the fluorescent signal was analyzed. (right) Plots of the mean intensity for the corresponding fluorescent signal going from the inside to the outside of the cell. Bars, $5 \mu \mathrm{m}$; time shown in seconds.
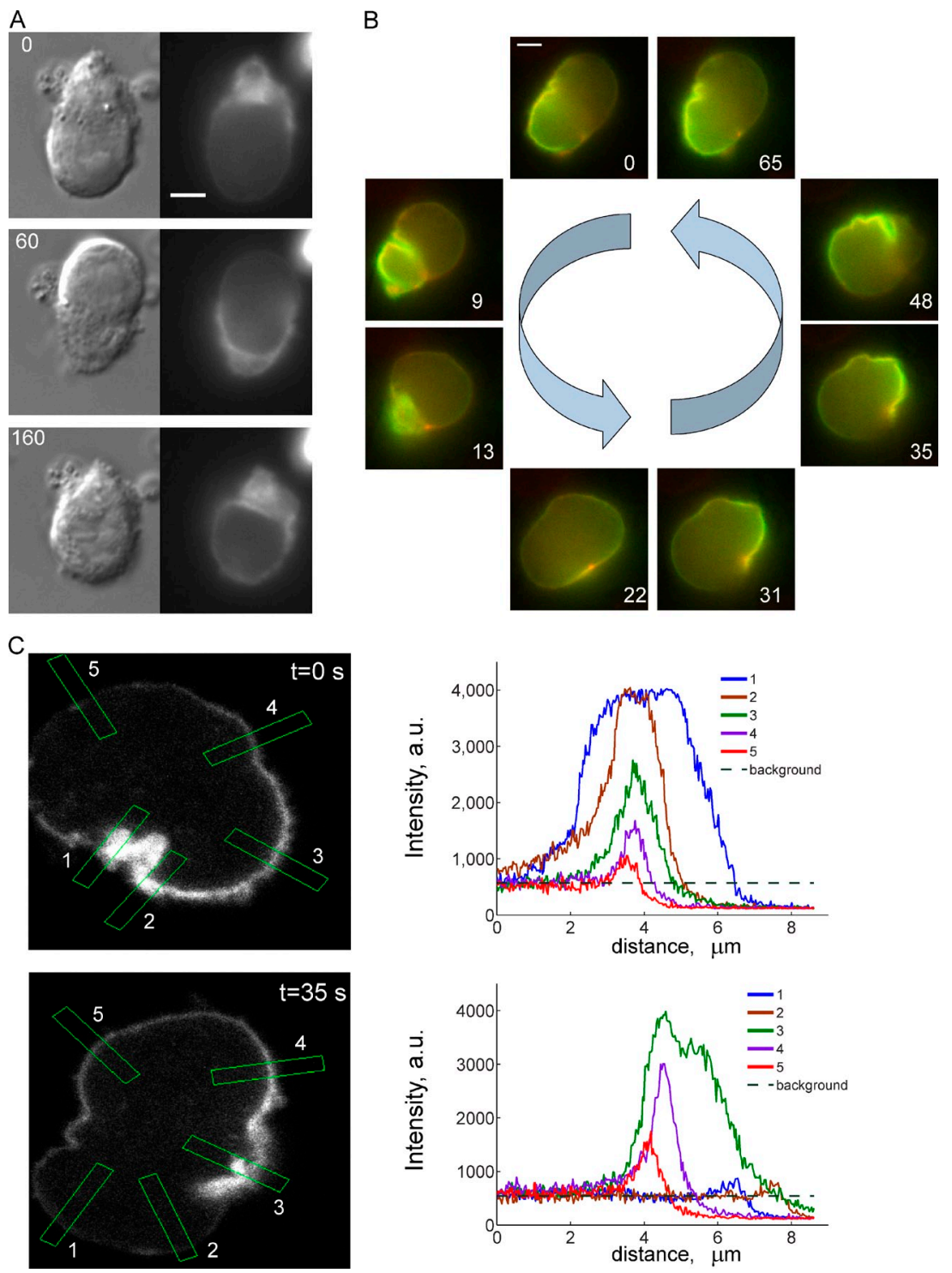

that the cyclic process of membrane-cortex compression and dilation generates a traveling wave of cortical actin density, which in turn generates oscillations in cell morphology and which, under proper environmental conditions, can produce amoeboid-like migration.

\section{Results}

\section{Cortical dynamics in living cells during periodic protrusions}

To examine cortical dynamics in living cells during oscillations, we used CHO cells that stably express Lifeact-GFP, which labels F-actin structures (Riedl et al., 2008). Time-lapse imaging using differential interference contrast (DIC) and epifluorescence shows how the morphology and actin cortex concurrently change during oscillations (Fig. 1 A). Fig. 1 B presents one complete period of the oscillatory phenotype and demonstrates the location and density of the highly polarized F-actin and myo$\sin$ in the cortex. Note the striking similarity in the F-actin and myosin distributions at the beginning $(t=0)$ and at the end of the period ( $t=65 \mathrm{~s}$; Video 1 ). This highly periodic behavior, often lasting several hours, indicates that the protrusions are a mechanochemically regulated process and not driven by stochastic fluctuations.

The intensity of the fluorescence signal is proportional to the number of fluorescently labeled molecules and provides a reliable estimate of relative density distribution. Fig. $1 \mathrm{C}$ presents examples of the cortical F-actin distribution estimated from the Lifeact-GFP fluorescence signal at several positions around the cell perimeter for two time points. Indeed, the main characteristic of these oscillating cells $(n=365)$ is the significant differences in the intensity and breadth of the fluorescent signal, originating in the F-actin-containing cortex, around the cell periphery. The density of the F-actin cortex, as judged by the intensity and breadth of the F-actin signal, can vary by up to an order of magnitude in different regions of cell or in the same region at different times. The breadth of the signal, presumably reflecting cortical thickness, can range from 0.3 to $4 \mu \mathrm{m}$ in different regions of cell periphery. For example, at $t=35 \mathrm{~s}$, the position labeled 1 is the most dense part of the cell cortex, 
A

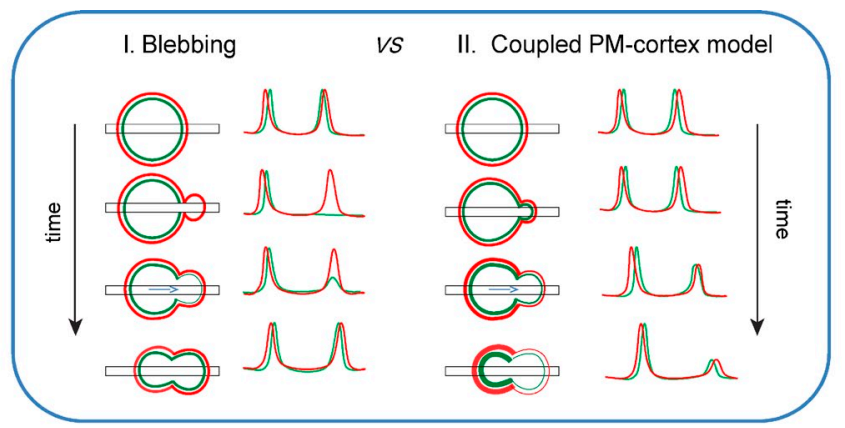

B
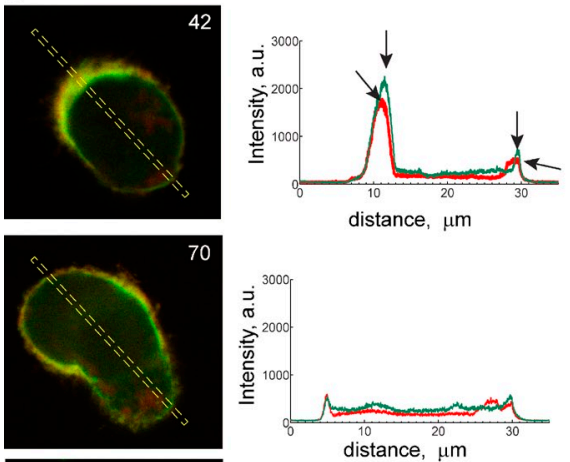

112
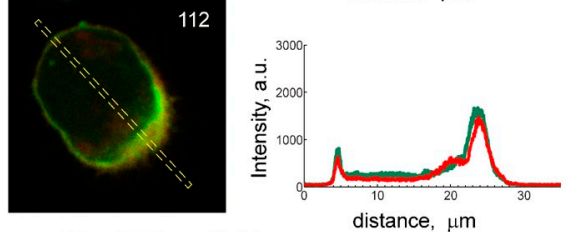

distance, $\mu \mathrm{m}$
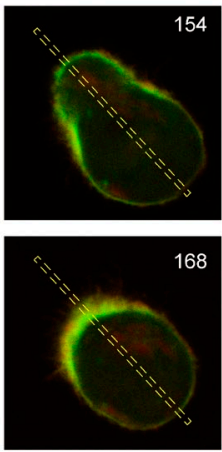

C

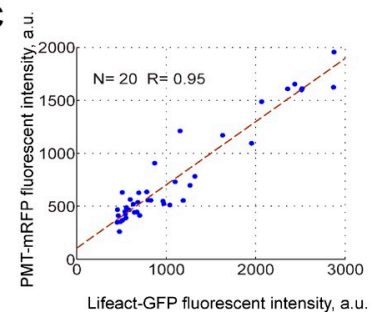

Figure 2. Cytoskeletal and PM coordination during periodic protrusions. (A) Predictions of fluorescent signal outcome based on two different models for rounded protrusions. Schematic drawing presents cell top view (left) and plot of fluorescent signals within the rectangle (right) of cortical F-actin (green) and PM (red). (B) Time-lapse confocal fluorescent images of a cell transfected by Lifeact-GFP (green) and PMT-mRFP (red; Video 2). The yellow rectangles $(33 \times 1 \mu \mathrm{m})$ show the positions where the fluorescent signal was analyzed. To the right of the images are plots of averaged line scans of fluorescence intensity going from the left to the right. (C) Correlation plot between maximum intensity of F-actin and PM fluorescence at the cell periphery presented in $B$ (correlation coefficient $R=0.95$ ). In total, $n=31$ cells from 12 experiments were analyzed. For each cell, 20 consecutive frames were analyzed and each frame provides two positions for correlation analysis where the rectangle intersects the cell margin; the arrows in B (top right) denote examples of chosen positions (vertical arrows for cortex; oblique arrows for PM). Time shown in seconds. whereas at $t=0$ it was the least dense. In contrast, in nonoscillating cells, the F-actin cortex is much more evenly distributed and stable in time (Fig. S1).

\section{The fluorescent signals associated with the} PM and F-actin cortex are highly correlated Previously, it was suggested that periodic protrusions of rounded cell bodies are bleb-like in nature and that this mechanism is used for "blebby" amoeboid migration (Paluch et al., 2005; Charras and Paluch, 2008; Fackler and Grosse, 2008). During blebbing, the PM detaches from the cortex in some stochastically chosen position and creates a large, cortex-free protrusion as a result of cytosolic pressure. Subsequently, the actin cortex redevelops under the bleb and retracts the bleb. We asked if the periodic protrusions formed in the oscillatory phenotype are initially cortex-free or whether, in fact, the membrane-cortex interface remains coupled.

The expectations for both mechanisms are described schematically in Fig. 2 A. If we monitor the fluorescent signals of both F-actin and the PM within a narrow rectangle placed on the cell, the resting, rounded cell, at its periphery, will show similar values for the cortex and PM, respectively, at any azimuth (Fig. 2 A, top row). During bleb initiation (Fig. 2 A. I), when the cortex breaks or the PM detaches from the intact cortex and expands, the F-actin should rapidly disappear in the vicinity of the blebbing PM and the PM signal would be expected to be visible without the corresponding cortical F-actin signal. When the cortex redevelops to support the bleb, a weak F-actin signal should appear again. As the protrusion expands, the F-actin signal will grow together with protrusion size until the developing cortex becomes strong enough to resist cytosol pressure, thereby preventing further expansion. Eventually, cortical development will be completed and the F-actin signal will return to its initial value. Therefore, the PM and F-actin fluorescent signals will not be correlated during bleb development.

As an alternative, we could hypothesize that the PM and cortex remain as a couple and simultaneously undergo coordinated shape changes so that the PM is supported by at least a thin F-actin-containing cortex at all times (Fig. 2 A, II). In this case, the result of an imaging experiment will be qualitatively different from the bleb mechanism: the signals from F-actin and PM will become progressively weaker during protrusion growth and stronger as the protrusion retracts. Therefore, in all stages of protrusion, the PM and F-actin signals will be highly correlated. Thus, in principle, we can distinguish between the two hypotheses by analyzing the fluorescent signals during the protrusion process.

We examined experimentally the simultaneous behavior of both the cortex and the PM by transfecting cells stably expressing Lifeact-GFP with a PM target monomeric red fluorescent protein (PMT-mRFP; $n=22$ cells) or ErbB2-mRFP ( $n=9$ cells), an expressed membrane protein (Liu et al., 2007; Video 2 
Figure 3. Periodic protrusions are initiated by detachment and rounding. Cells spread for $24 \mathrm{~h}$ and trypsinized on the microscope stage during the timelapse recording (Video 3). (A) Composite images of DIC and Lifeact-GFP fluorescence. Media was replaced with trypsin at time 0 . After $\sim 90 \mathrm{~s}$ trypsin was replaced with media to prevent complete detachment. (B) DIC and corresponding fluorescent image from region denoted by yellow rectangle (A) for two oscillation cycles that appeared immediately after cell rounding. (C) Kymographs generated at the positions and directions given by the red and purple dotted arrows shown in $\mathrm{A}$. The positions of $\mathrm{T} 1$ and $\mathrm{T} 2$ show the time when trypsin was added (T1) and when trypsin was replaced by media (T2). Two red angles (I and II) show the slope of kymograph reflecting the speed of retraction during the final stage of cell rounding (I) and during the retraction of a protrusion (III). The top kymograph is from a cell transfected with Lifeact-GFP, whereas the bottom one is from a nontransfected cell. Images present one experiment out of 15 repeats. (D and E) Comparison of surface area in spread and rounded cell. Schematic (D) compares the size of a sphere required to accommodate all the cell surface from the spread state $(R=24.5 \mu \mathrm{m})$ to the actual size of the rounded state $(r=10.2 \mu \mathrm{m})$.

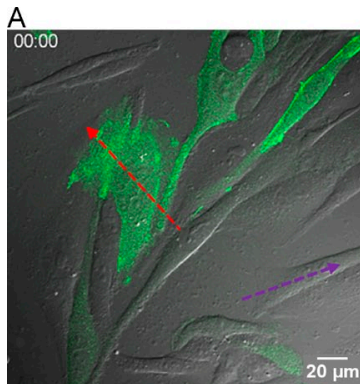

B
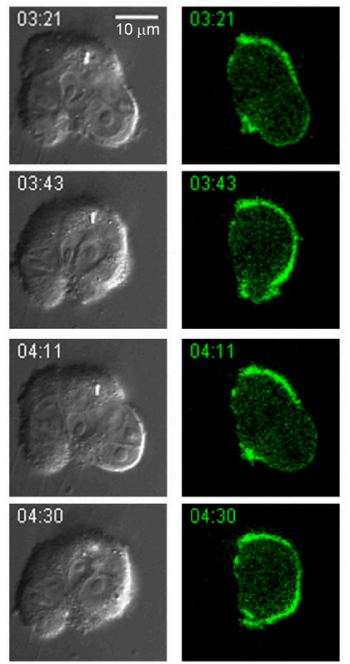

D

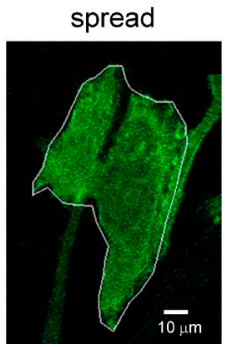

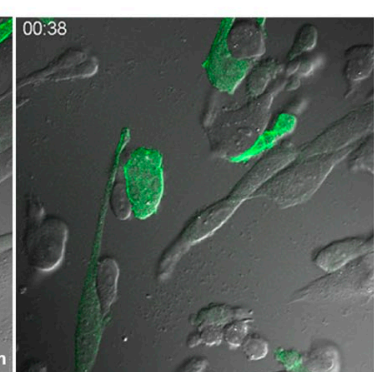

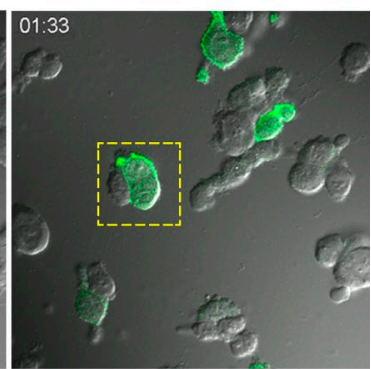

C
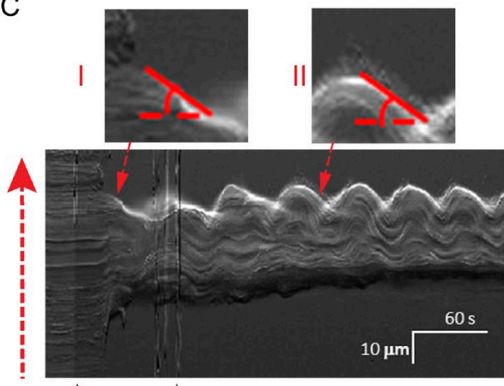

$\begin{array}{cc}\uparrow & \uparrow \\ \text { T1 } & \text { T2 }\end{array}$

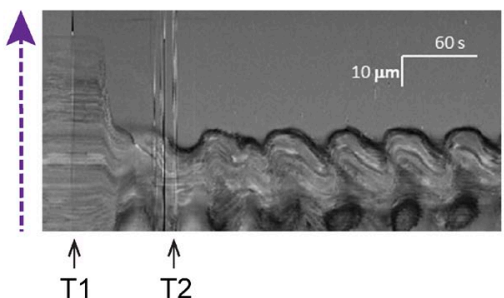

E
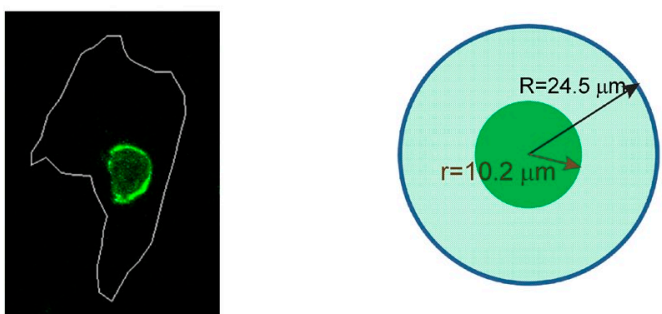

and Fig. 2 B). Note the strong correlation between cortical actin and PM signals during oscillations and the fact that the apparent thickness of PM and cortex signals are also highly correlated. When the PM on one side of the cell has protruded and the image shows apparent thinning of the PM fluorescent signal, the underlying cortex also appears thinner. This behavior is quantified using a scatter plot (Fig. 2 C) for fluorescence intensities of cortical F-actin versus PM signals for the cell presented in Fig. 2 B. The signals on the cell margin are highly coordinated in time and space with a correlation coefficient of $\mathrm{R}=0.95$. The mean correlation coefficient for 31 analyzed cells with two different membrane probes from 12 different experiments is $\mathrm{R}=0.856 \pm 0.076(\mathrm{SD})$. It is also important that a decrease in signal intensity is correlated with a protrusion expansion and an increase of signal corresponds to a protrusion retraction (Fig. 2 B, 70 vs. 112 s). Analyses and graphs for the PM-cortex distribution along the cell perimeter for an oscillating as contrasted to a nonoscillating cell are presented in Fig. S1. The strong correlation between the intensity and shape of two fluorescent signals means that the PM and cortex are simultaneously undergoing the same changes during all phases of the periodic protrusive phenotype.

As we reported previously (Costigliola et al., 2010), in a rounded cell population, many cells oscillate (50-80\%), whereas other cells may be inactive or undergo substantial blebbing. We analyzed the fluorescent signal from a subpopulation of cells that underwent blebbing (Fig. S2). When these blebs initially grow, the signal from membrane is bright and without an accompanying F-actin signal. Within the next several seconds, the F-actin signal grows substantially and reaches a maximum after $\sim 15-20 \mathrm{~s}$, whereas the membrane signal stays constant or slightly decreases. This dynamic blebbing is local, small scale, and apparently stochastic; in contrast, cells undergoing cortical oscillations exhibit large-scale changes that are highly periodic with a predictable position for the shape transformation.

To address the question of whether true blebbing might be occurring on a faster time scale than that presented in Fig. 2, we used spinning disk confocal microscopy to increase our time resolution by acquiring an image of Lifeact-GFP every $100 \mathrm{~ms}$ (Video 3). Kymographs $(n=19)$ were constructed along a line 
across the periodically protruding cells and they show the periodic nature of protrusion and retraction that is characteristic for this phenotype (Fig. S3). Importantly, the cortex shape evolves smoothly and the fluorescent signal does not show visible breaks. Kymographs $(n=9)$ were also constructed for cells labeled for the PM and F-actin and they also show consistent correlation between the PM and F-actin signals (Fig. S3). Thus, using this optical resolution on the 100-ms time scale, we do not observe separation of cortex and membrane. Of course, we cannot guarantee that some breakage of the F-actin-containing cortex does not occur occasionally but it does not appear to be a major characteristic of the phenotype we are describing.

\section{Initiation of the periodic protrusive phenotype and cortical folding \\ by cell rounding}

We asked how the oscillations previously observed in many cell types (Pletjushkina et al., 2001; Paluch et al., 2005; Salbreux et al., 2007; Costigliola et al., 2010) are initiated. We imaged spread cells detaching from the substrate after treatment with trypsin. Note that this experiment was performed without deliberately depolymerizing microtubules. Surprisingly strong periodic protrusions appear as the final phase of cell rounding (Fig. 3, A-C; and Video 4). The kymograph in Fig. $3 \mathrm{C}$ shows no delay between the consecutive processes of cell rounding and the onset of the oscillatory phenotype; moreover, the speed of cell retraction at the end of rounding is similar to the speed of protrusion retractions during oscillations (Fig. $3 \mathrm{C}$, I and II, see the comparison of the kymograph slopes at the two positions).

We analyzed the surface area of cells in the spread and rounded states. During cell rounding, the apparent surface area of the cell decreases from 2.5-fold up to 8-fold, requiring the excess surface to be compacted in $<1$ min (Fig. 3, D and E). We estimated that the mean decrease in surface area for 33 cells from seven different experiments was $\sim 4.3 \pm 1.6$ (SD)-fold. On this time scale, compaction of the PM by folding must be the predominant process as opposed to endocytosis, which takes much longer for this extent of surface interiorization (Steinman et al., 1983). Indeed, the PM after cell rounding is highly convoluted (Price, 1967; Furcht and Wendelschafer-Crabb, 1978). Because in the spread cell, the PM is supported with a thin layer of underlying cortex between 50- and 200-nm thick (Pesen and Hoh, 2005), it is reasonable to assume that during rounding the underlying cortex does not detach from the PM but, together with the PM, forms folds. Apparently, this shape transformation, which involves a large compaction of the PM-cortex couple, is sufficient to initiate the oscillatory phenotype with a period and amplitude similar to our previous observations (Kapustina et al., 2008; Costigliola et al., 2010). As the rounded protrusion develops, the PM-cortex layer unfolds to provide the additional surface required to cover the protrusion. The process of retraction simply folds back the PM and cortex in a manner similar to the mechanism used for cell rounding.

The presence of folds on the periphery of cells undergoing periodic protrusions was confirmed by transmission electron microscopy (TEM; Fig. 4, A and B; and Fig. S4). Note that in the text we refer the compaction of the membrane-cortex couple
A

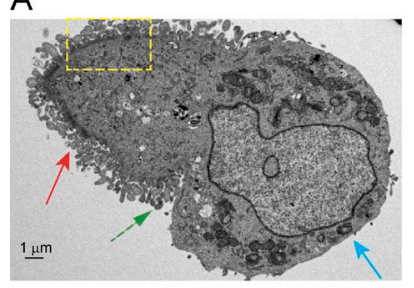

C

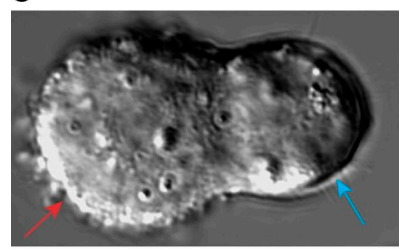

B

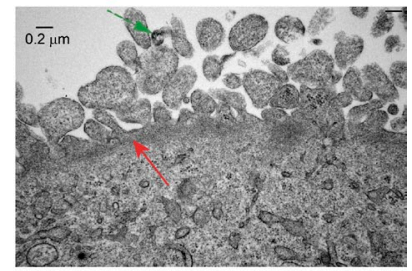

D

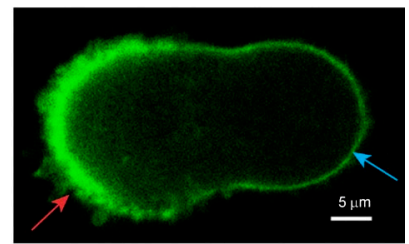

$\mathrm{E}$

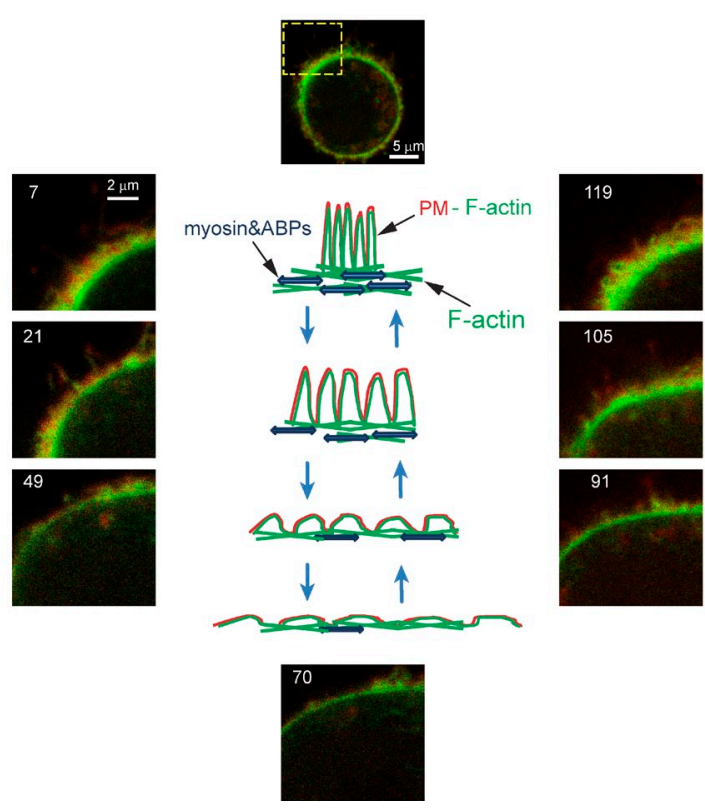

Figure 4. Folding of the PM-cortex layer in TEM. (A and B) TEM of a cell fixed during oscillations. Yellow rectangle $(A)$ shows the position where the magnified image (B) was taken. (C and D) Visual similarities between TEM and DIC (C) and confocal fluorescent images (D, Lifeact-GFP) of oscillating $\mathrm{CHO}$ cells. Red arrows point to dense cortical regions in the TEM image (A and $B$ ) and presumed membrane folds in the DIC image $(C)$ that are correlated with the dense, thick F-actin cortex in the confocal fluorescent image (D). Blue arrows point to smooth areas, lacking folds on cell periphery in TEM and DIC images (A and C), respectively, and a thinner cortical F-actin layer in the confocal fluorescent image (D). (E) Schematic view of PM-cortex folding and unfolding (middle) and corresponding confocal fluorescent images of the PM (red) and F-actin (green). The yellow rectangle in the top panel $(t=0)$ shows the position of the rest of the images (Video 5).

as folding; however, what appears as folding in 2D confocal images is really an array of finger-like or rounded projections when imaged at the ultrastructural level. It can be observed on the TEM image that one region of the cell margin shows folds that may appear as closed compartments in the thin section, whereas the distal regions of the cell appear smooth, corresponding to the protruding region (Fig. 4, A and B). The height of the folds varied from $20 \mathrm{~nm}$ to nearly $1 \mu \mathrm{m}$ and they also varied considerably in width. The region of densely compacted folds correlates with the position of denser material, presumably the underlying cortex. It also has visual similarities with 
Figure 5. Cortex periodic structure and estimation of membrane-cortex compaction. (A-D) An example of periodic structure, probably representing tightly packed folds of the PM (red) and F-actin (green) couple, is visible in the cortex of a rounded nonoscillating cell $(n=8)$. (A) Folds at lower magnification. (B) Folds at high magnification from box in $A$. ( $C$ and D) Line scan of F-actin and PM mean fluorescence intensity along a line $(5.5 \times 0.2 \mu \mathrm{m})$ indicated (bottom) from box in A. (E-I) Estimation of degree of membrane-cortex compaction based on F-actin fluorescence intensity and membrane movement relative to obvious fiduciary marks. (E and G) Merged $\mathrm{DIC}$ and $\mathrm{F}$-actin fluorescent images at two time points. ( $\mathrm{F}$ and $\mathrm{H})$ F-actin fluorescent images at high magnification from box in $\mathrm{E}$ and $\mathrm{H}$. The yellow band indicates where the mean fluorescence intensity and perimeter length were determined. At $t=0$, the segment of the perimeter, which later became a protrusion, was $18.2 \mu \mathrm{m}$ long with a mean intensity of 1,864 \pm 7,02 arbitrary units (a.u.). After $16 \mathrm{~s}$, this densely folded region became a protrusion bounded by a perimeter segment of $32.0 \mu \mathrm{m}$ with a mean intensity of $828 \pm 476$ a.u. (l) Plot of normalized changes of the perimeter length of protrusion and corresponding changes in mean fluorescence intensity for $n=$ 17 protrusions from 12 examined cells.
A

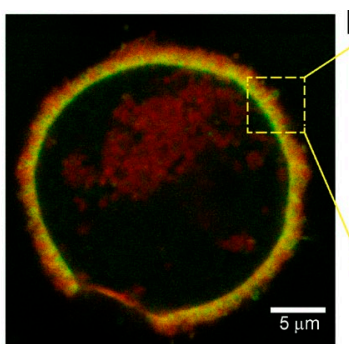

C

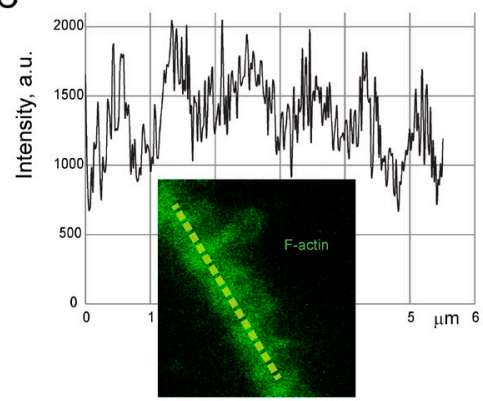

E

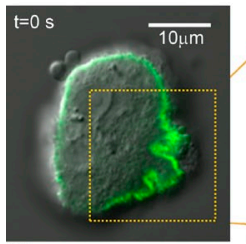

G

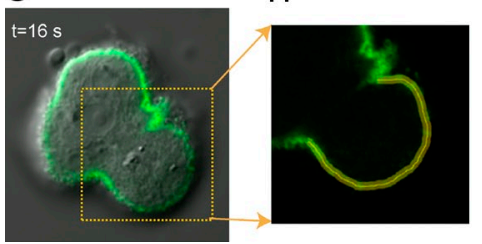

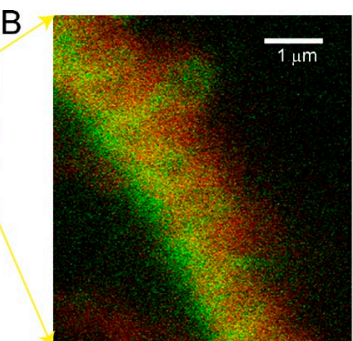

$\mathrm{D}$

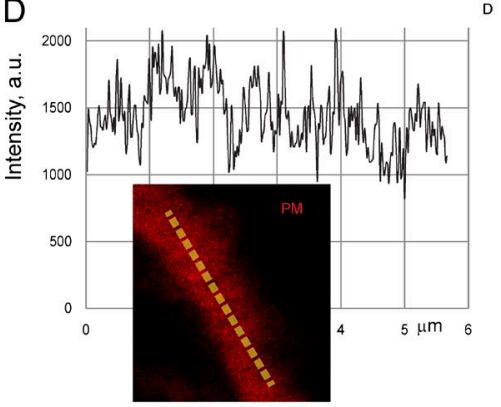

I

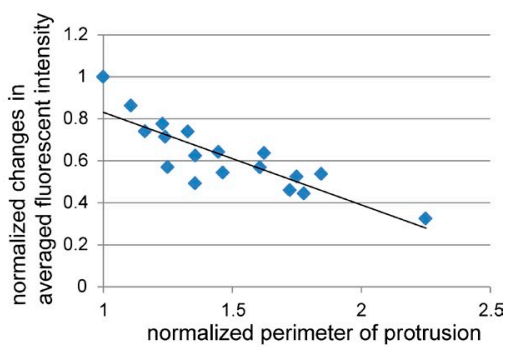

the appearance of membrane folds visible in DIC and fluorescent imaging (Fig. 4, C and D). The folds are always filled with cytoplasmic material and sometimes small organelles are trapped inside them (Fig. S4), indicating that they are not typical blebs. The majority of small folds cannot be visualized by traditional optical methods because of their density and size. When folds are compressed and dense they appear in the fluorescence image as a thick and wide band on the cell margin. When the cortex relaxes and releases highly compacted folds, they become more visible in the optical section (Video 5 and Fig. 4 E).

Our data suggests the coupled PM-cortex compression and dilation mechanism depicted in Fig. 4 E (middle). This hypothesis states that the PM and cortex remain coupled in all phases of the dynamic protrusions exhibited by rounded cells and that these protrusions occur via an unfolding of the PMcortex composite layer. In the first frame $(t=7 \mathrm{~s})$, the image depicts the situation when the PM and cortex have a dense and compact structure (width of $\sim 1.7 \mu \mathrm{m}$ ) that originates from PMcortex folds as described schematically in the middle panel of Fig. 4 E (top). We assume these folds are kept intact by the underlying actin-myosin network (Cai et al., 2010), which is cross-linked by actin binding proteins (Doherty and McMahon, 2008; Kasza et al., 2009; Michelot and Drubin, 2011). When the activity of myosin and actin binding proteins decreases, the folds are released and the PM-cortex couple relaxes and dilates because of cytosolic pressure. An image in the same location at a later time (42 s) shows a substantially thinner PM-cortex layer signal with decreased intensity. At later times $(t=77 \mathrm{~s})$, the PM and cortex fluorescent signals are fully dilated, having an apparent width of $\sim 0.3 \mu \mathrm{m}$. After this stage, the process of contraction and folding occurs, bringing the cell margin back to its initial state $(t=119 \mathrm{~s})$ in which the cortex and PM signals are both wider and more intense (Video 5).

Surprisingly, the periodic structure (presumably folding) of the PM-cortex couple in rounded but nonoscillating cells is also visible in Fig. 5 (A-D), suggesting that properly packed, evenly distributed folds could stabilize cell shape and prevent the oscillatory phenotype.

We estimated the degree of membrane-cortex compaction using several different measurements. From the DIC and fluorescent images, we measured the perimeter length and mean fluorescence intensity inside a 1- $\mu \mathrm{m}$-wide band along the perimeter of protrusion (Fig. 5, E-I). The segment length was measured based on membrane movement relative to obvious fiduciary marks, visible on the DIC image. We made similar measurements using dual membrane (PMT-mRFP) and F-actin (Lifeact-GFP) fluorescent signals measured in a $2-\mu \mathrm{m}$-wide band along the whole perimeter (Fig. S5 A). The plot in Fig. 5 I 

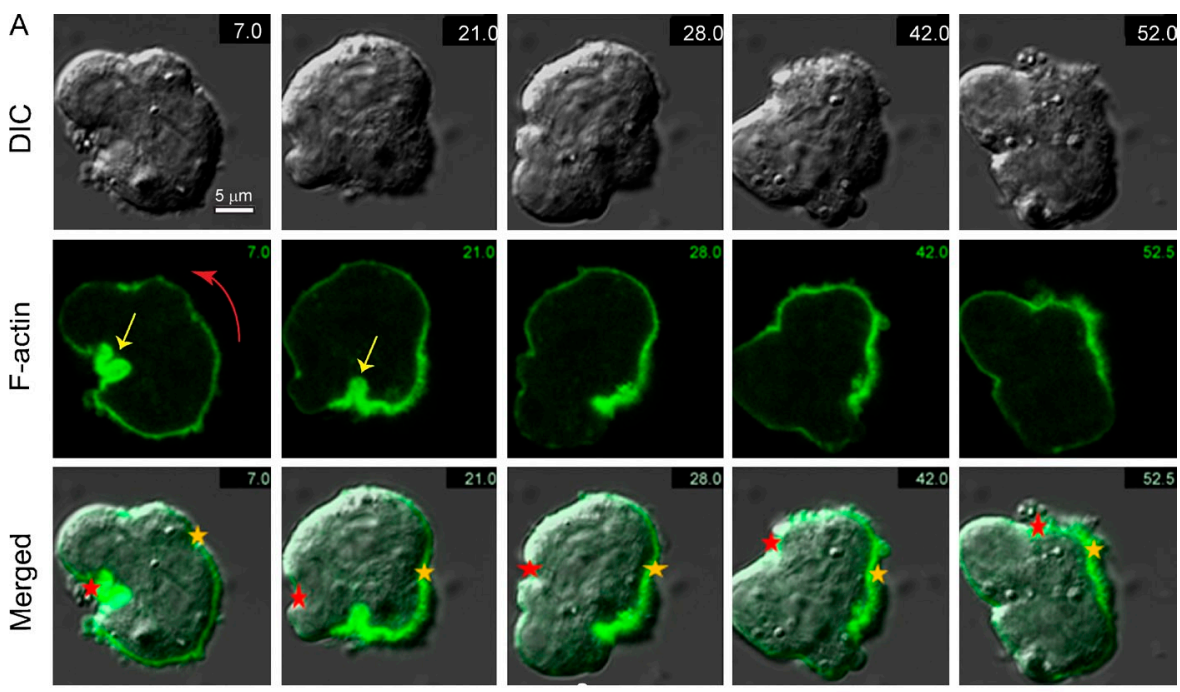

B
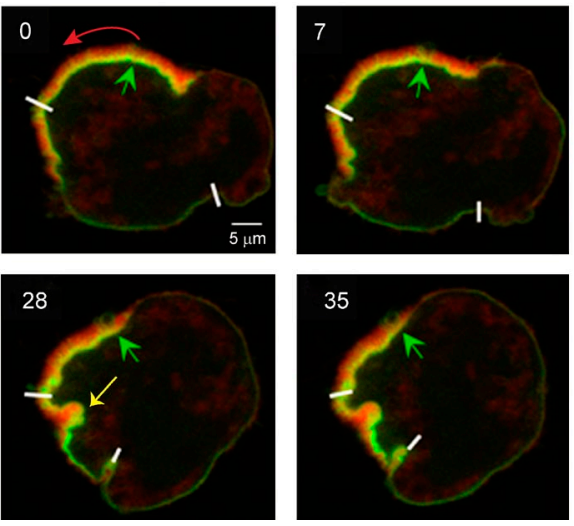
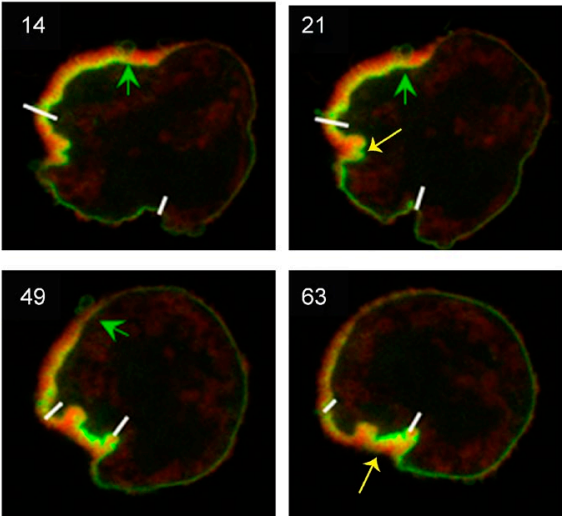

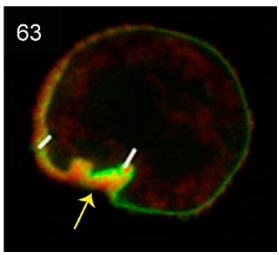

Figure 6. A traveling wave of cortical actin density generates periodic protrusions and large secondary folds. (A) Time lapse of DIC and F-actin confocal fluorescent images of cells undergoing periodic protrusions. Red arrows show the direction of the cortical wave propagation. The stars are two fiduciary markers that show how the cell periphery apparently becomes dilated (21 s) and compressed (52 s) during the periodic protrusive cycle. (B) Time lapse of merged F-actin (green) and PM (red) confocal fluorescent images. Two white bars are fiduciary markers to illustrate the process of producing large accordion-like folds (Video 6). Green arrows point to a partially unfolded region in the middle of the density wave at $t=0$ that moves toward the relaxed region $(t=63 \mathrm{~s})$. Yellow arrows point to a large-scale structure on the cell periphery. demonstrates that the changes in mean intensity of the cortex underlying a protrusion are inversely proportional to the changes in the perimeter length of the protrusion. From this analysis we can estimate that $\sim 80-85 \%$ of changes in cortical intensity are accounted for by cortex folding alone. The remaining changes in intensity could be caused by F-actin polymerization/ depolymerization and/or optical effects caused by the cortex obliquely intersecting the confocal section.

We also analyzed the distribution of intensity values for both PM and F-actin fluorescent signals measured around the cell perimeter ( $n=15$; Fig. S5 A) and calculated the mean of the lowest $10 \%$ of the intensity values and the mean of the highest $10 \%$ of the intensity values. Assuming that the lowest intensity represents the intensity/unit length of unfolded membranecortex and the highest values represent the intensity/unit length of folded membrane-cortex, we estimated the compression factor for the membrane-cortex couple. This analysis suggests that membrane-cortex folding results in a compression factor between two and six relative to an unfolded cell surface. The result of these calculations also indicated that the compression factors for membrane and cortex are similar.

Next we approximated the amount of membrane compaction from TEM images ( $n=12$; Fig. S4) by measuring a contour length that includes folds and compared it to one that does not. This estimation indicates that cells are capable of storing up to
6.5 times more surface in folds compared with an unfolded surface, which is in agreement with the analysis of fluorescent intensity and also with our estimate of how much the apparent cell surface decreases during cell rounding.

\section{PM-cortical actin traveling waves}

The compression and release of the folded PM-cortex often creates a traveling wave where contractility in one region of the cell periphery is constantly compacting and folding the excess PM-cortex couple, which was created by dilation in a distal portion of the periphery (Fig. 6 A). This wave of cortical density travels along the cell periphery for several hours creating a stable oscillatory pattern. We can estimate the velocity of wave propagation as $\sim 1 \mu \mathrm{m} / \mathrm{s}$, considering that the mean cell radius is $\sim 10 \mu \mathrm{m}$ and mean traveling wave period is $\sim 60 \mathrm{~s}$.

Despite the visual illusion that the dense portion of cortex moves around the cell periphery, no mass is actually being transported; rather, a compression wave is propagated with new folds in the membrane-cortex layer generated at the leading edge of the wave that unfold at its trailing edge. This process can be seen by monitoring particular reference points on the cell periphery during the oscillatory phenotype, identified on the companion DIC images (Fig. 6 A). Red and yellow stars on Fig. 6 A (merged) identify such fiduciary markers; by monitoring their relative positions, we appreciate the extent of membrane extension 

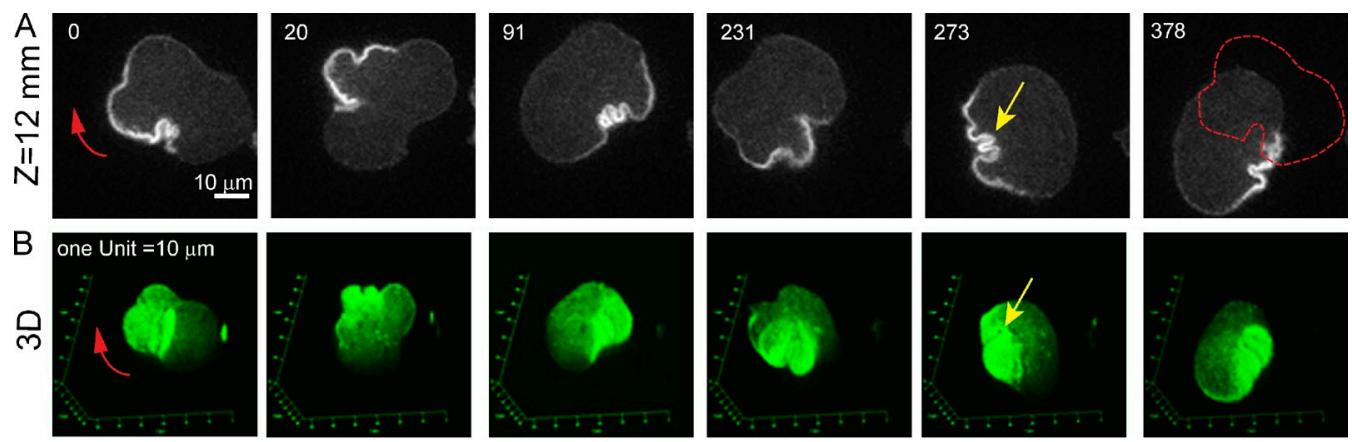

C
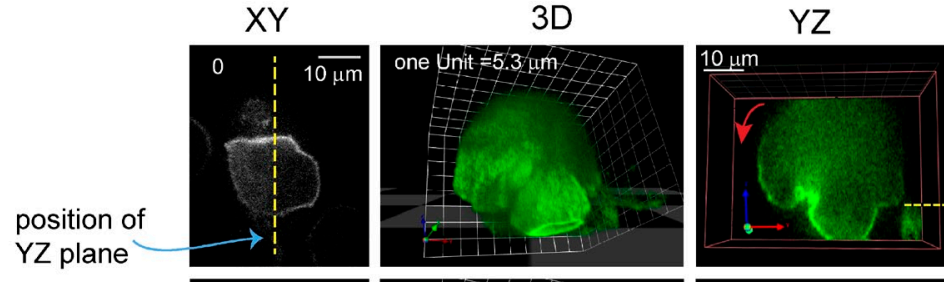

K position of
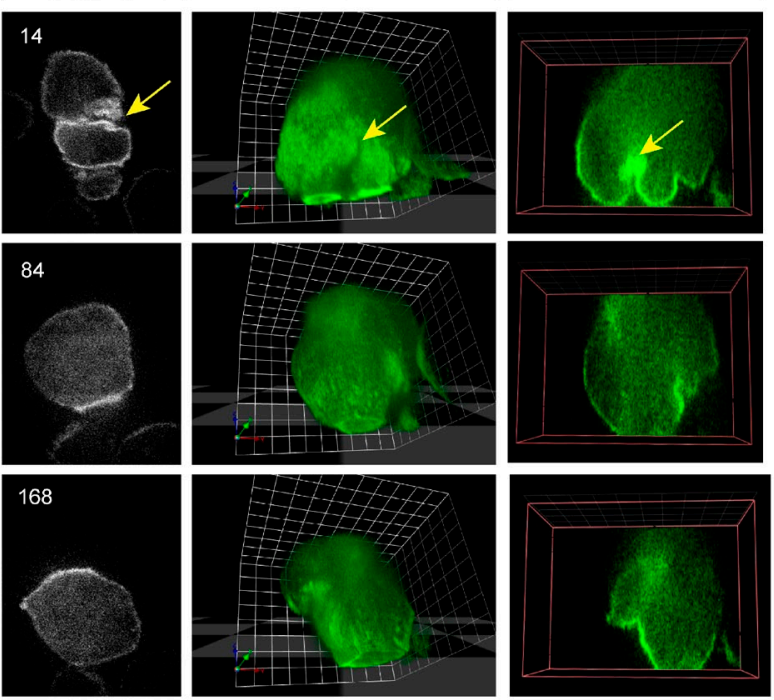

D

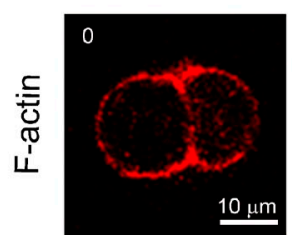

Cortex polarization before blebbistatin

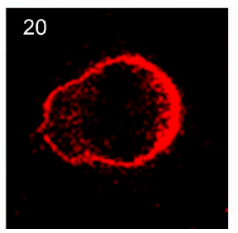

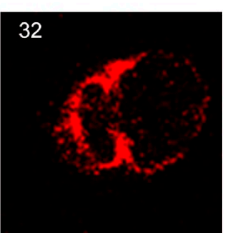

$140 \mathrm{~s}$ after $\mathrm{Bb}$

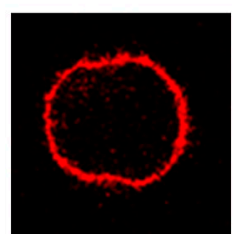

Figure 7. A wave of cortical density depends on myosin contractility and can travel in different directions. (A) Time lapse of a single XY single plane $(Z=12 \mu \mathrm{m})$ from a $Z$ stack of F-actin confocal fluorescent images; the red outline indicates the initial position. Red arrows show the direction of cortical wave propagation, visible on both XY and 3D images. Yellow arrows point to the position of large folds that in 3D (B) look like deep clefs. (B) Corresponding 3D reconstruction of cell cortex built from a Z stack of confocal images (Video 7). (C) Traveling wave of cortex density is not always visible in the confocal plane. Simultaneous views of 3D cell cortex reconstruction (middle), corresponding confocal image at position $Z=8 \mu \mathrm{m}$ from the substrate (left), and reconstructed image of $Y Z$ orthogonal projection (right). On XY confocal images, the propagation of density traveling wave is not visible, whereas on the YZ orthogonal projection (red arrow) the traveling wave is apparent (Video 8). (D) Confocal fluorescent images obtained near the mid-plane of the cell for three time points demonstrate polarization of F-actin cortex (Lifeact-RFP) during periodic protrusion and resultant equilibration of cortex density and thickness after inhibition of myosin II with $5 \mu \mathrm{M}$ blebbistatin. This effect is reversed by illumination in blue light (Video 9). Time shown in seconds.

and folding that occurs in periodic protrusions. In particular, note that between 7 and $21 \mathrm{~s}$, the membrane-cortex couple near the red star unfolds at the trailing edge of the wave and becomes a rounded protrusion. This protrusion further extends between 21 and $42 \mathrm{~s}$, during which time the distal region of the couple refolds to its maximum extent at $52 \mathrm{~s}$ as shown by the minimal separation of the of fiduciary markers.

Similar evidence for the folding dynamics that lead to wave propagation is shown in Fig. $6 \mathrm{~B}$, where a distinct compression of the membrane-cortex couple can be seen during the 


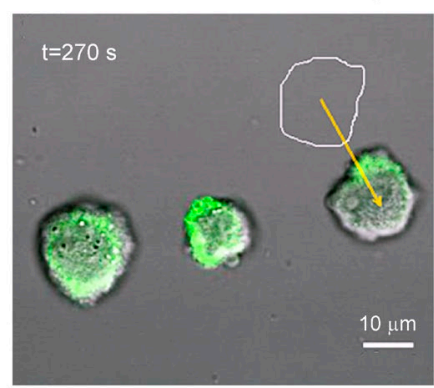

Migration of $\mathrm{CHO}$ cells on collagen
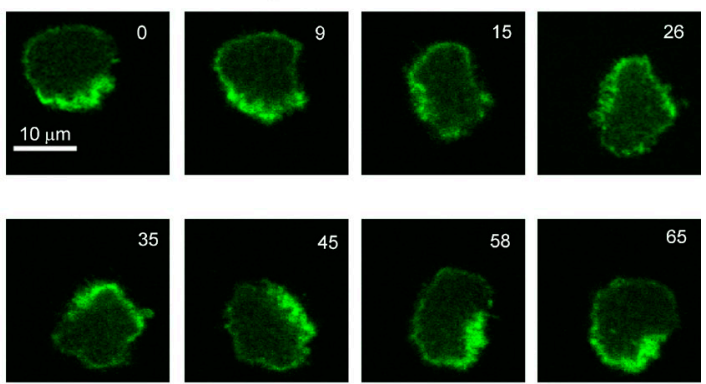

B
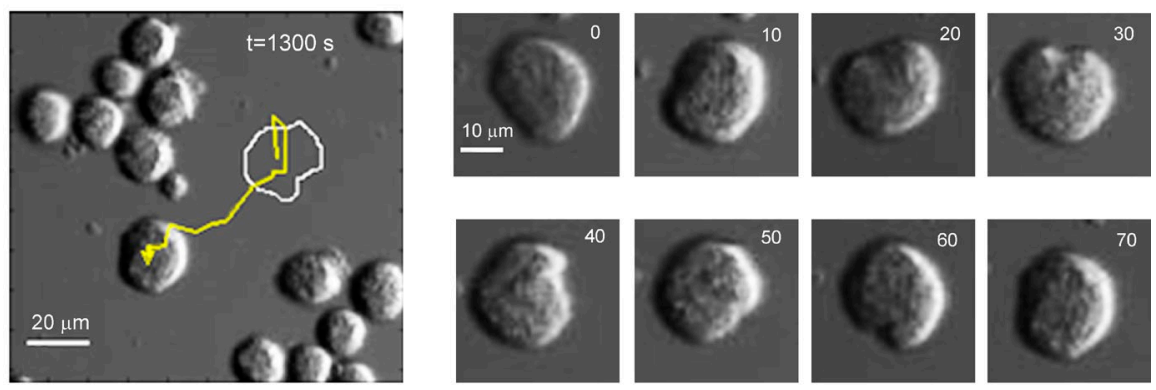

C

Migration of rounded $\mathrm{NIH} 3 \mathrm{~T} 3$ cells on glass
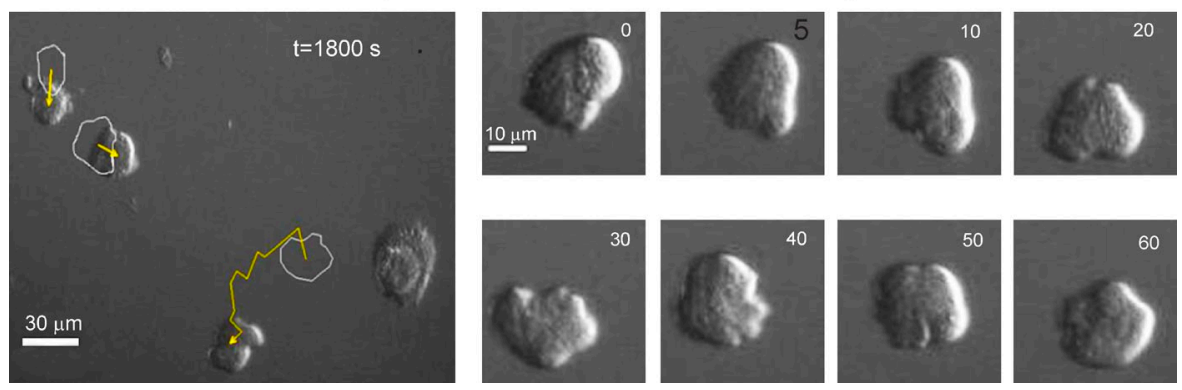

Figure 8. Periodically protruding cells can migrate. Left panels show start positions (white outline) and final positions of migrating cells and paths of cell movement (yellow). The separate images in the right panels illustrate the coordination between periodic protrusion and cell translocation for one oscillation period. (A) Merged DIC and Lifeact-GFP fluorescent images of rounded $\mathrm{CHO}$ cells without artificially depolymerized microtubules plated on collagen $(10 \mathrm{mg} / \mathrm{ml})$. Net translocation is $22 \mu \mathrm{m}$. (B) DIC images of rounded MDA-MB cells plated on glass with depolymerized microtubules. Total path is $92 \mu \mathrm{m}$ and net translocation is $62 \mu \mathrm{m}$. (C) DIC images of rounded NIH 3T3 cells plated on glass with depolymerized microtubules. Total path is $108 \mu \mathrm{m}$ and net translocation is $70 \mu \mathrm{m}$. progressive folding from 0 to $63 \mathrm{~s}$ and the accompanying decrease in separation of the two white bars (Fig. $6 \mathrm{~B}$ and Video 6).

The strong PM-cortex compression frequently forms a large-scale structure on the cell periphery (Figs. 6 and 7, yellow arrows), which in the confocal plane appears as an invagination and can be part of a traveling contractile ring often seen on the surface of oscillating cells (Fig.1, A and B; and Videos 1, 2, and 6; Paluch et al., 2005; Salbreux et al., 2007; Costigliola et al., 2010). In 3D reconstructions (Fig. 7, B and C; and Video 7), these topological structures look like deep clefts on the cell surface. It is important to note that a traveling wave is not always apparent in confocal sections because a section may transect the cell such that the periodic propagating wave is partially missed. For example, when a traveling wave propagates in a direction normal to the substrate, it can be visualized in the orthogonal (YZ) views built from the set of $\mathrm{Z}$ section confocal planes (Fig. $7 \mathrm{C}$ and Video 8). Sometimes not one but several small traveling waves are visible on the surface of the oscillating cell. The spatial relationship between the confocal plane and the direction of the propagating waves generates diversity in the images of the periodic protrusive phenotype.

The primary driver for these morphological oscillations must reside within the cortical cytoskeleton. Indeed, inhibition of conventional myosin by blebbistatin completely abolishes the protrusive phenotype and spatially equilibrates cortical density (Fig. 7 D). Moreover, consistent with the fact that blebbistatin activity can be photoinhibited, restoring myosin contractility, the oscillation phenotype was rescued by illumination with blue light, resulting in a nearly identical period and similar shape dynamics as observed before myosin inhibition (Video 9). This result indicates the importance of myosin II-mediated contractility in developing of polarized distribution of F-actin cortex. We expect that many other actin binding proteins may, of course, be involved in providing structure and regulation to the cortex and periodic protrusions (Dai et al., 1999; Tseng et al., 2001; Kruchten and McNiven, 2006; Courson and Rock, 2010; Gilden and Krummel, 2010; Michelot and Drubin, 2011; Strehle et al., 2011).

\section{Periodically protruding cells perform amoeboid-like migration}

Strikingly, we observed that cells can translocate while undergoing periodic protrusions (Figs. 8 and 9 and Video 10), suggesting that this behavior is relevant to cell migration. We investigated the conditions under which periodically protruding cells migrate, anticipating that such migration would be abrogated by strong 
Figure 9. Random and directional migration of cells that are periodically protruding. (A) Random migration of rounded $\mathrm{CHO}$ cells plated on serum-coated glass under agar with intact microtubules. Yellow outlines show cell position at the beginning of recording and arrows denote the direction and net migration distance. The mean speed was $0.53 \pm 0.15 \mu \mathrm{m} / \mathrm{min}$. (B) Bar graph representing the distribution of cell directions within $1-h$ migration of $\mathrm{CHO}$ cells under agar with " $\mathrm{O}$ " indicating the direction along the $X$ axis. The plot was calculated from two separate recordings for a single experiment out of two repeats ( $n=65$ cells). (C) Migration of NIH 3T3 cells under agar with chemoattractant (200 ng/ml PDGF) presented in a distal well in the agar gel. Green arrows point in the direction of the chemoattractant source. The initial position of the cells is outlined in yellow and red, where yellow outlines indicate cells moving in an amoeboid-like fashion and red outlines indicate cells migrating mesenchymally. Mean speed of amoeboid-like cells was $0.55 \pm 0.14 \mu \mathrm{m} / \mathrm{min}$. (D) Bar graph representing the distribution of cell directions $1 \mathrm{~h}$ after plating under agar with " $\mathrm{O}$ " indicating the direction toward to the well containing chemoattractant. The plot was calculated for $n=51$ cells from three separate experiments.
A

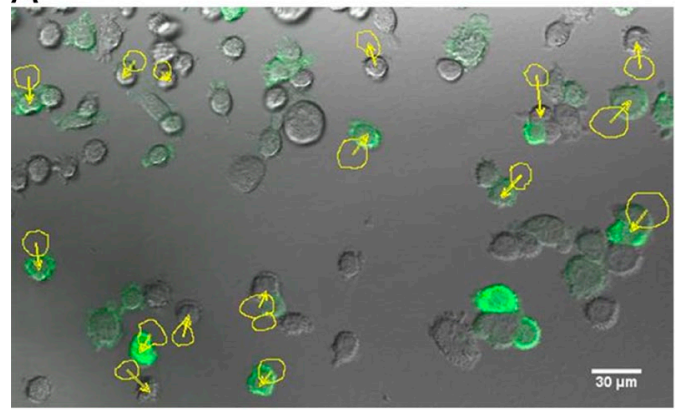

C

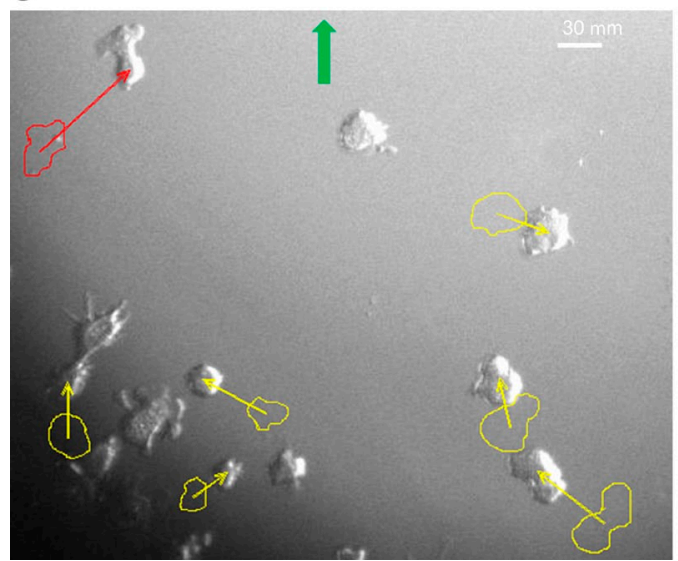

B

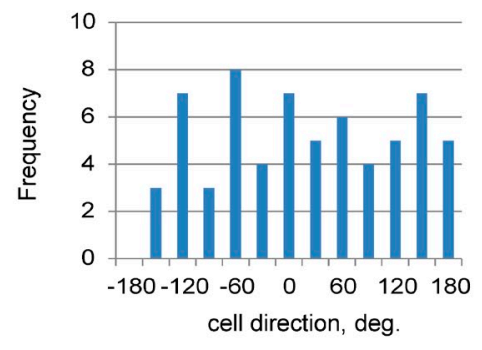

D

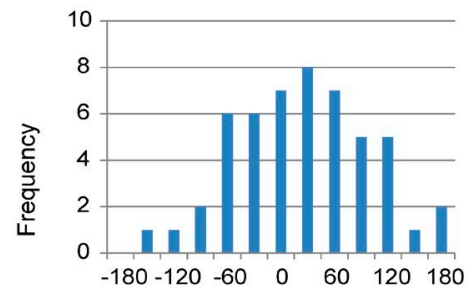

cell direction with respect to direction of chemotactic source, deg. adhesion to the substratum. Indeed, $\mathrm{CHO}$ cells plated on glass bottom dishes develop sufficiently strong adhesions so that net migration rarely ensues during oscillations (Videos 1-5). In contrast, $\mathrm{CHO}$ cells plated on collagen $(10 \mathrm{mg} / \mathrm{ml})$ can undergo robust migration (Fig. $8 \mathrm{~A}$, mean speed of $4.9 \mu \mathrm{m} / \mathrm{min}$ ). Other cell types can also undergo this type of migration soon after plating. Fig. 8 (B and C) shows that rounded, periodically protruding mammary carcinoma cells, MDA-MB, and NIH 3T3 fibroblasts with depolymerized microtubules undergo amoeboid-like migration before they fully spread on glass bottom dishes. The migrations covered $92 \mu \mathrm{m}$ at a mean speed of $4.2 \mu \mathrm{m} / \mathrm{min}$ for MDA-MB cells and $108 \mu \mathrm{m}$ at a mean speed of $3.6 \mu \mathrm{m} / \mathrm{min}$ for NIH 3T3 cells. Experimentally, it is difficult to avoid some initial convective flows in the chamber on these length scales; here we present data after these flows have dissipated as judged by the lack of directed movement of naturally occurring fiduciary markers.

We asked whether periodically protruding cells could undergo directed migration toward a chemoattractant source. To accomplish this, we used the under agar chemotactic assay (Nelson et al., 1975; Heit and Kubes, 2003; Bergert et al., 2012). If $\mathrm{CHO}$ cells are sandwiched between an agar slab and a serumcoated substratum, without chemoattractant, they will undergo amoeboid-like migration in random directions, albeit at slower speeds (Fig. 9, A and B). Fig. 9 (C and D) shows that when NIH 3T3 cells are presented with PDGF in a distal well within the agar gel both rounded, periodically protruding cells and already spread cells migrated toward the source of the chemoattractant. During the movement several cells were constantly switching mode of migration from amoeboid-like to mesenchymal and back. After the first hour, $67 \%$ of the migrating cells have directions that lie within a $120^{\circ}$ cone centered on the chemoattractant source. Thus, in general, this type of amoeboid-like migration in which the cells acquire speeds ranging from 0.5 to $5 \mu \mathrm{m} / \mathrm{min}$ requires the cells to be rounded and to have an optimum adhesiveness. Moreover, this type of migration can apparently be directed by chemoattractants.

\section{Discussion}

We have shown that the morphological oscillations of rounded cells result from compression and dilation of the coupled membrane-cortex layer. The PM has a limited capacity for areal extension $(<4 \%)$ before rupture (Sheetz et al., 2006) but a high degree of plasticity with a low bending energy (Lipowsky, 1991; Farsad and De Camilli, 2003), which makes it a good candidate for folding. The actin cortex is capable of supporting a variety of shapes (Michelot and Drubin, 2011), it being a dynamic, signalingregulated structure where myosin and other actin binding proteins determine the network architecture. We demonstrated for the first time that the periodic protrusive phenotype results from membrane-cortical actin traveling waves. The observation of traveling cortical waves is novel and may represent, for example, how rounded cells choose directionally in response to chemoattractants. The cortical wave may also be analogous to actin wave propagation in the lamellae of leukocytes or fibroblasts (Dunn and Zicha, 1995; Servant et al., 1999; Driscoll et al., 2012).

We envision that the cortical actin traveling wave plays at least two important roles in regulating net migration of a periodically protruding cell. First, the traveling wave established 


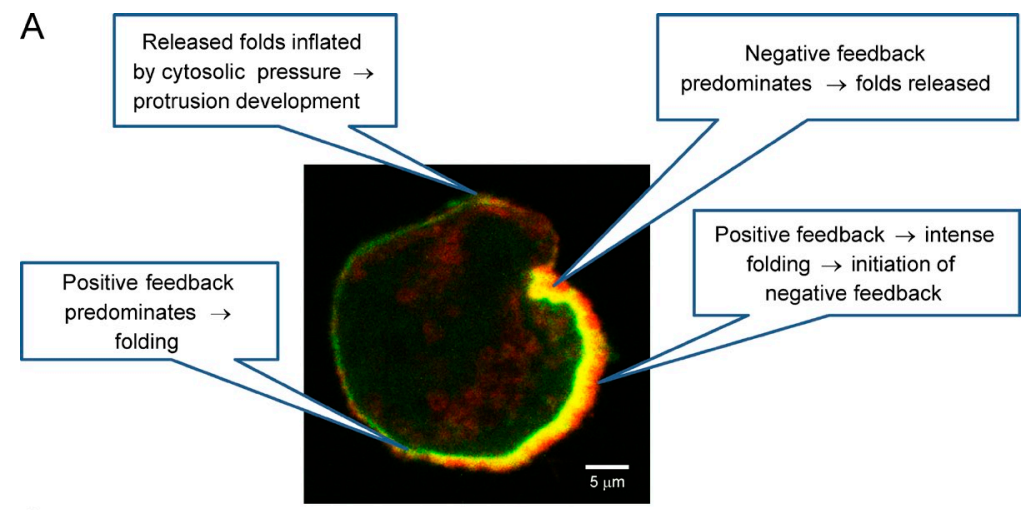

Figure 10. Schematic of hypothetical mechanisms for the traveling wave that leads to periodic protrusions. (A) Postulated locations on the periphery of a cell exhibiting a cortical actin traveling wave where feedbacks and folding dynamics occur. (B) Schematic representations of coordination between traveling wave, folding, protrusions, attachment, and migration. See Discussion for further explanation.

B

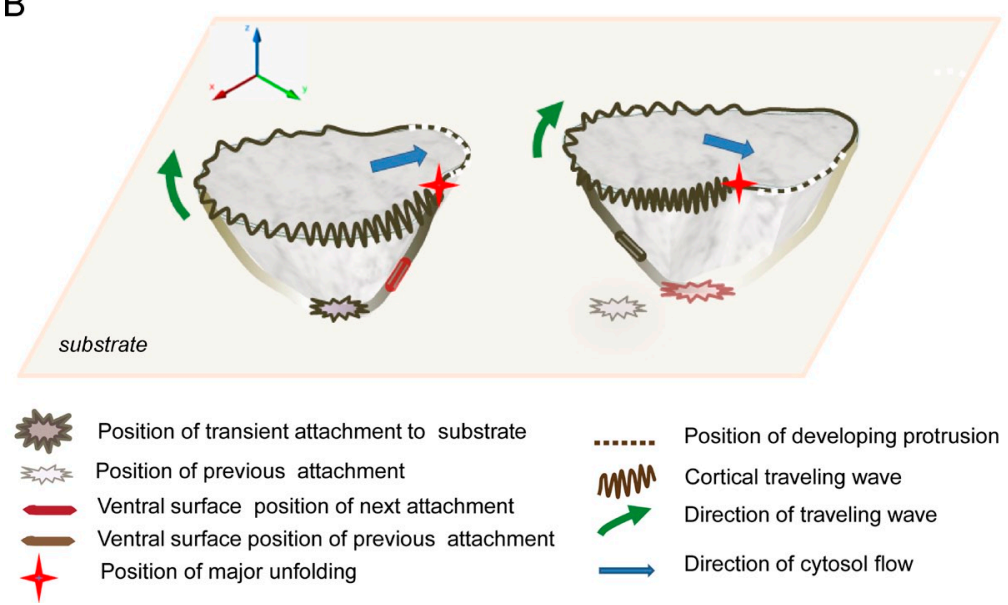

the position of the future protrusion by releasing the membranecortex layer from the heavily folded region (Fig. 10). Second, the traveling wave directs the cytosol flow used to inflate the protrusion by transiently positioning the contractile region of the cortex that drives the flow; this flow, in turn, determines the speed and, to a certain extent, the size of the protrusion.

Fig. 10 presents a schematic view for how the cortical traveling wave may be related to net cell migration. If there is sufficient adhesion in the central region of the cell proximal to the contractile region to promote traction, the rounded protrusion, inflated by cytosolic flow, will move the center of mass of the cell in the direction of the protrusion (Fig. 10 B, left). Subsequent movement of the contractile wave may result in rupture of the initial adhesive region but for migration to be sustained another region of transient adhesion must be established forward of the initial adhesion (Fig. 10 B, right). Note that the cartoon in Fig. $10 \mathrm{~B}$ represents the case when the traveling wave propagates in the direction parallel to the substrate. In reality, cortical waves can travel in any fixed direction along the cell periphery, which may necessitate a modification of our simple cartoon view. For example, if the wave is traveling in the plane normal to the substrate, it would create visual similarity with the "walking" cell (Fig. 7 and Video 8).

For periodic protrusions to result in net migration, cell adhesions to the substrate need to be dynamic and coordinated with the movement of the protrusion. Without sufficient adhesion, the cortical traveling wave will propagate around the periphery without producing net migration and if cells are too adherent, they will oscillate without net displacement.
Additionally, to create a directionality, some form of cell polarity must be established either externally (chemotactic/ haptotactic gradient) or internally (for example, by asymmetrically distributed adhesions or cortical flexibility; Lorentzen et al., 2011).

How might the cortical traveling wave be propagated in terms of the signaling pathways? Microtubule status clearly plays a role in the oscillatory phenotype as deliberately depolymerizing them increases the amplitude of the oscillations (Costigliola et al., 2010). Whether this is caused solely by the release of guanine nucleotide exchange factor $\mathrm{H} 1$ to activate RhoA or caused also by a structural effect remains to be determined. In general, traveling waves require positive feedback and some form of negative feedback. We assume that a positive feedback loop operates globally to increase cortical density and folding until a local negative feedback mechanism rapidly dissipates cortical density and folding. These loops are hypothesized to be initiated through phosphoinositide metabolism in the regions depicted schematically in Fig. 10 A. Indeed, our preliminary studies with inhibitors of PI3K and PLC (Wortmannin, PI3K-IV, and U73122) indicate an important role for inositol lipids processed by these enzymes. In general, phosphoinositides provide regulation of the subcellular localization of many scaffolding and actin severing proteins that are involved in the interplay between the actin cytoskeleton and PM. In turn, these proteins can provide feedback from the actin cortex to the phosphoinositides and regulate their spatial distribution through restricted diffusion or sequestration (Sheetz et al., 2006; Hilgemann, 2007; Golebiewska et al., 2008). Thus, spatiotemporal regulation of the membrane concentration of phosphoinositides 
might produce the control required to sustain the periodic protrusive phenotype. This regulation would be coupled to the RhoA-MLCK-MLCP-myosin II pathway, known regulators of the oscillatory phenotype, via guanine nucleotide exchange factors and GTPase activating proteins.

The notion of compression and dilation of the membrane-cortex couple has several antecedants. It was previously suggested that cells use surface, reserved in membrane "protuberances," for spreading and initiating cell locomotion (Erickson and Trinkaus, 1976; Gauthier et al., 2011). The migration of fibroblasts was shown to require "retraction-induced spreading" (Chen, 1981), whereby excess cell surface accumulated during tail retraction was stored in dorsal folds, which relaxed when the cell underwent the next round of lamellipodial spreading.

Periodically protruding cells show several characteristics that are remarkably similar to cells undergoing the blebby-type amoeboid migration in which rounded protrusions prevail. 3D computer reconstructions of Lifeact-GFP fluorescence (Fig. 7 and Videos 7 and 8) also showed morphological similarity to the blebby type of amoeboid migration (Mierke et al., 2008; Friedl and Wolf, 2009). In addition, both phenotypes lack strong focal adhesions and stress fibers that pull on the substrate to generate movement (Friedl and Wolf, 2010). Both have been shown to be regulated by the phosphoinositides (Vemuri et al., 1996; Saarikangas et al., 2010) and the Rho family of small GTPases, in particular RhoA, plays a major role, whereas Rac appears to be less important (Sahai and Marshall, 2003; Costigliola et al., 2010; Paňková et al., 2010). The fact that periodically protruding cells can migrate adds credence to this phenomenon being a model for the blebby type of amoeboid locomotion that is tractable from both experimental and theoretical points of view (Wang et al., 2012).

We presume that compression-dilation and classical blebbing are two distinct mechanisms that may be related in the following way. Membrane-cortex relaxation and unfolding is a global cell process to be contrasted to more local blebbing (Charras and Paluch, 2008; Bergert et al., 2012). The process of compression-dilation requires a highly flexible and elastic cortex, capable of rapid and global transformation and it is likely that microtubule depolymerization is one of the possible ways to achieve this flexibility. In contrast, if the cortical cytoskeleton is more rigid, global shape transformation through the compression-dilation mechanism would be less likely but blebbing could still occur.

The compression-dilation mechanism provides a simple but efficient way to transform cell shape and offers the advantage that the position of the transformation or protrusion can be spatiotemporally regulated in a precise fashion. In addition, in both developmental and metastatic cancer contexts, amoeboidlike cells must negotiate the interstices of the filamentous structures composed of extracellular matrix proteins. Cell movement through these structures in all likelihood requires greater structural strength than would be furnished by an initially unsupported PM bleb but could be provided by the protrusive PM being supported by a subjacent cortical layer. We anticipate that the cortical compression-dilation hypothesis will find applicability not only for cell migration but for various types of cell shape transformations, including those involved in cell division, differentiation, and spreading

\section{Materials and methods}

\section{Cell culture and transfections}

$\mathrm{CHO}$ and $\mathrm{NIH} 3 \mathrm{~T} 3$ fibroblast cell lines were obtained from University of North Carolina Lineberger Comprehensive Cancer Center Tissue Culture facility. A human breast carcinoma, MDA-MB, cell line was gift from C. Huang (University of Kentucky, Lexington, KY). MDA-MB and NIH 3T3 cells were grown in DMEM medium containing 10\% FBS (Gibco), $100 \mathrm{U} / \mathrm{ml}$ penicillin/streptomycin, and $4 \mathrm{mM}$ l-glutamine. $\mathrm{CHO}$ cells stably expressing Lifeact-GFP (the small 17-aa peptide, Lifeact, fused to GFP) and expressing myosin regulatory light chain fused to RFP (MLC-RFP) were obtain using the lentivirus expression vector pLL5.0 (backbone pLL3.7) and were a gift from the James Bear laboratory (University of North Carolina at Chapel Hill, Chapel Hill, NC). CHO cells were grown in DMEM/F1 2 medium containing $10 \% \mathrm{FBS}, 100 \mathrm{U} / \mathrm{ml}$ penicillin/streptomycin, and $4 \mathrm{mM} \mathrm{L-glutamine}$ (Gibco) for $2 \mathrm{~d}$ to confluency and trypsinized with trypsin-EDTA. The supernatant was then spun down at $<1,000 \mathrm{rpm}$ and the cells were resuspended in $5 \mathrm{ml}$ of media. $1 \mathrm{ml}$ of this suspension was then added to 35-mm (MatTek Corporation) glass bottom dishes, along with an additional $1.5 \mathrm{ml}$ of media. $0.2 \mu \mathrm{M}$ colchicine (Sigma-Aldrich) was added at the time of cell plating unless otherwise noted. The Trypsin-EDTA solution (SigmaAldrich) was used for experiments involving cell detachment. Blebbistatin (Sigma-Aldrich), Wortmannin (LC Laboratories), and U73122 (Tocris Bioscience) were used for inhibition experiments.

For the PM visualization, we transiently expressed the PMT-mRFP or ErbB2 transmembrane protein fused to RFP (ErbB2-mRFP) in CHO cells that were stably expressing Lifeact-GFP. Construction of the PMT-mRFP and ErbB2-mRFP was described elsewhere (Liv et al., 2007). PMT is a lipidlinked protein that resides almost exclusively on the inner leaflet of the PM. The PMT sequence encodes the first 15 aa of the X-linked retinitis pigmentosa protein RP2 (Chapple et al., 2002), which contains a consensus myristoylation site and a potential palmitoylation site. The PMT sequence was fused to the $N$ terminus of mRFP through an overlap extension PCR strategy, and the PMT-mRFP fragment was inserted into $\mathrm{pEGFP-N1}$ vector (Takara Bio Inc.) through Agel and Notl sites. The ErbB2 is a transmembrane protein from the growth factor receptor tyrosine kinases family. The mRFP gene was amplified from pRSETB/mRFP vector and fused to the $C$ terminus of the full-length human ErbB2 through an overlap extension PCR strategy. The ErbB2-mRFP fragment was inserted into pcDNA 3.1/Zeo vector through Afel and Notl sites. PMT-mRFP and ErbB2-mRFP constructs were generously provided by Ichiro Maruyama (Genome Institute of Singapore, Singapore).

$\mathrm{CHO}$ cells were transiently transfected by PMT-mRFP or ErbB2-mRFP using Lipofectamine Plus reagent (Invitrogen) and images were taken 24-48 $\mathrm{h}$ after transfection.

Agarose gel was made from powder (UltraPure Agarose; Invitrogen) using cell culture media with 10\% FBS (Nelson et al., 1975; Heit and Kubes, 2003). Agarose medium was delivered to $35-\mathrm{mm}$ glass bottom dishes and allowed to harden. To perform a migration experiment, two 4-mm holes were made in the agar gel and these holes were separated by $10 \mathrm{~mm}$. Cells suspended in DMEM media $\left(30 \mu \mathrm{l}, 10^{7} \mathrm{cells} / \mathrm{ml}\right)$ with depolymerized microtubules $(0.2 \mu \mathrm{M}$ colchicine) were placed in one well and PDGF $(30 \mu l, 200 \mathrm{ng} / \mathrm{ml}$ in DPBS$)$ in the other well for chemotactic experiments.

\section{TEM}

$\mathrm{CHO}$ cells treated with colchicine as described in the previous paragraph were plated on 60-mm Nunc Permanox dishes and visualized under the FluoView 1000 microscope (Olympus) to ensure the presence of oscillations. Oscillating cells were fixed in 3\% glutaraldehyde/HBSS, $\mathrm{pH} 7.4$, at $37^{\circ} \mathrm{C}$ for $15 \mathrm{~min}$ followed by $1 \mathrm{~h}$ at RT. After three rinses with $0.15 \mathrm{M}$ sodium phosphate buffer, $\mathrm{pH} 7.4$, the monolayers were postfixed with potassium ferrocyanide-reduced osmium for $1 \mathrm{~h}$ at RT ( $1 \%$ osmium tetroxide/ $1.25 \%$ potassium ferrocyanide/0.15 M sodium phosphate buffer). After washes in deionized water, the cells were dehydrated using increasing concentrations of ethanol $(30,50,75,100$, and $100 \%$ for $10 \mathrm{~min}$ each) followed by embedment in Polybed 812 epoxy resin (Polysciences, Inc.). The cells were sectioned en face by a diamond knife. Ultrathin sections $(70 \mathrm{~nm})$ were stained with $4 \%$ aqueous uranyl acetate for 15 min on 200 mesh copper grids followed by Reynolds' lead citrate $11.33 \mathrm{~g}$ lead nitrate, $1.76 \mathrm{~g}$ 
sodium citrate, and $30 \mathrm{ml}$ distilled water) staining (Reynolds, 1963). Samples were viewed using a LEO EM910 transmission electron microscope (Carl Zeiss), operating at an accelerating voltage of $80 \mathrm{kV}$. Digital images were taken using a charge coupled device (CCD) camera (Orius SC 1000) and DigitalMicrograph 3.11.0 software (Gatan, Inc.).

\section{Microscope image acquisition}

The optical imaging for this investigation was performed using several microscope systems. All images were obtained in cell culture media using an environmental chamber control, the temperature was maintained at $37^{\circ} \mathrm{C}$ and a $5 \% \mathrm{CO}_{2}$ solution was perfused into the chamber. Images in Fig. 1 A were acquired using an IX-81 inverted microscope (Olympus). Images were taken at $60 \times$ with a SensiCam QE CCD camera (Cooke Corporation) using MetaMorph 8.0 software. Images in Fig. 1 B and Video 1 were obtained on a Nipkow-type spinning disk confocal scan head (CSU-10; Yokogawa Corporation of America) with a $60 \times 1.45$ NA objective and 1,024 × 1,024 camera (Andor Technology). Images in Fig. $7(A-C)$ and Videos 7 and 8 were acquired using a Cell Observer Spinning Disk (AxioObserver Z1; Carl Zeiss) with a CSU Photometrics Evolve $512 \times 512$ camera (Yokogawa Corporation of America), $63 \times / 1.40$ objective, and stage top incubation. Images were recorded and processed using Axiovision version 4.8.2 software. Fig. S3 and Video 3 were acquired using an Andor Revolution XD Spinning Disk Confocal (Olympus) with an iXon3 $512 \times 512$ camera, a $60 \times / 1.40$ objective, and MetaMorph 8.0 software.

Images in Figs. 8 ( $\mathrm{B}$ and $\mathrm{C}$ ) and 9 and Video 10 were acquired using a VivaView Incubator Fluorescence Microscope (Olympus) with an Orca R2 cooled CCD camera (Hamamatsu Photonics) and MetaMorph 8.0 software. The dish containing the gel assembly was placed in a VivaView incubator (Olympus) and DIC images at the edge of the cell well and in a direction toward the chemoattractant well were recorded every 5 to $10 \mathrm{~s}$ for $4 \mathrm{~h}$ using a UPLSAPO 10x objective.

The rest of images and videos were acquired on the FluoView 1000 or FluoView FV1000MPE laser confocal scanning microscopes (Olympus) using LUMPLFL $60 \times$ W $\backslash I R$ NA:0.90, PLAPON $60 \times / 1.42$, and $100 \times / 1.40$ oil immersion objectives.

The imaging data were analyzed using ImageJ, Volocity, and Matlab software. Adobe Photoshop and Illustrator were used only for resizing and adjusting images for figure presentations.

\section{Quantification of fluorescent images}

We developed a Matlab (MathWorks) program to automate the analysis of isolated cells. The area of analysis was defined for each cell individually, as cells varied in size and shape. To minimize the effect of unequal photobleaching of two-color fluorescent signals, the analysis was limited to 20 consequent frames. For each frame, the value of mean intensity along the longer side of the rectangle was calculated. The width of the rectangle was equal to $1 \mathrm{\mu m}$. First, the positions and values of the intensity maximum on each side of the cell near the border were identified and measured for the F-actin fluorescence signal. Then the peak value for the PM fluorescent signal was selected as the maximum value inside the 500-nm interval around the position of corresponding F-actin signal maxima. The calculations were performed under visual control to verify proper positioning and absence of artifacts. The correlation coefficient and corresponding $p$-value were calculated using Matlab functions.

\section{Online supplemental material}

Fig. S1 shows the correlation between fluorescent signals from F-actin cortex and PM in several positions along the edge of an oscillating and a nonoscillating cell for two time points. Fig. S2 shows laser scanning microscope images of fluorescent signals from PM and F-cortex for a rounded $\mathrm{CHO}$ cell undergoing blebbing. Fig. S3 shows the kymographs constructed from images of Lifeact-GFP and PMT-RFP fluorescent signals taken on a Revolution XD spinning disk confocal microscope at 100-ms intervals. Fig. S4 shows TEM images of $\mathrm{CHO}$ rounded cells that were fixed during oscillations and $\mathrm{CHO}$ cells spread for $24 \mathrm{~h}$ on a plastic culture dish. Fig. S5 demonstrates an estimation for the degree of membrane-cortex compaction in folded regions. Video 1 demonstrates cytoskeletal dynamics during oscillation. Video 2 shows the coordination between the PM and F-actin dynamics during periodic cell protrusions. Video 3 shows oscillating cell cytoskeletal dynamics using fast acquisition (100 ms) on a spinning disk confocal microscope. Video 4 demonstrates that cell oscillations can be triggered by detachment from substrate and subsequent rounding. Video 5 shows the coordinated folding and unfolding of the PM- and F-actin-containing cortex. Video 6 shows a cortical traveling wave on the periphery of an oscillating cell. Video 7 presents the propagation of cortical actin traveling wave in $3 D$ reconstruction and a corresponding $X Y$ confocal plane. Video 8 shows a 3D reconstruction of the cell cortex and F-actin traveling wave viewed in a $Y Z$ orthogonal projection. Video 9 demonstrates the reversible loss of oscillatory behavior as a result of myosin inhibition by blebbistatin. Video 10 shows that rounded cells can translocate using periodic protrusions as a mechanism. Online supplemental material is available at http:// www.jcb.org/cgi/content/full/jcb.201204157/DC1.

We thank James Bear for generous provision of cells expressing cytoskeletal proteins and Ichiro Maruyama for providing constructs. We thank Nancy Costigliola, Keith Burridge, Richard Cheney, James Bear, and Cai Huang for discussions and critical reading of an earlier version of this manuscript. We also thank Ping Liu, Zaozao Chen, Michelle Itano, and Aaron Neumann for discussions and Vicky Madden for technical assistance in TEM studies. We express our gratitude to the University of North Carolina (UNC) Microscopy Services Laboratory, UNC-Olympus Imaging Research Center, and UNC Zeiss Imaging Center for technical support.

This work was carried out with financial support from the US Army Research Office (58306-MA), National Institutes of Health (GM078994), National Institutes of Health Cell Migration Consortium (U54 GM064346), and National Science Foundation (DMS-1200535).

Submitted: 30 April 2012

Accepted: 4 December 2012

\section{References}

Bergert, M., S.D. Chandradoss, R.A. Desai, and E. Paluch. 2012. Cell mechanics control rapid transitions between blebs and lamellipodia during migration. Proc. Natl. Acad. Sci. USA. 109:14434-14439. http://dx.doi.org/ 10.1073/pnas. 1207968109

Brill, J.A., R. Wong, and A. Wilde. 2011. Phosphoinositide function in cytokinesis. Curr. Biol. 21:R930-R934. http://dx.doi.org/10.1016/j.cub.2011.10.001

Cai, Y., O. Rossier, N.C. Gauthier, N. Biais, M.A. Fardin, X. Zhang, L.W. Miller, B. Ladoux, V.W. Cornish, and M.P. Sheetz. 2010. Cytoskeletal coherence requires myosin-IIA contractility. J. Cell Sci. 123:413-423. http://dx.doi.org/10.1242/jcs.058297

Chapple, J.P., A.J. Hardcastle, C. Grayson, K.R. Willison, and M.E. Cheetham. 2002. Delineation of the plasma membrane targeting domain of the Xlinked retinitis pigmentosa protein RP2. Invest. Ophthalmol. Vis. Sci. 43:2015-2020.

Charras, G., and E. Paluch. 2008. Blebs lead the way: how to migrate without lamellipodia. Nat. Rev. Mol. Cell Biol. 9:730-736. http://dx.doi .org/10.1038/nrm 2453

Chen, W.T. 1981. Surface changes during retraction-induced spreading of fibroblasts. J. Cell Sci. 49:1-13.

Costigliola, N., M.T. Kapustina, G.E. Weinreb, A. Monteith, Z. Rajfur, T.C. Elston, and K. Jacobson. 2010. RhoA regulates calcium-independent periodic contractions of the cell cortex. Biophys. J. 99:1053-1063. http://dx.doi.org/10.1016/j.bpj.2010.06.010

Courson, D.S., and R.S. Rock. 2010. Actin cross-link assembly and disassembly mechanics for $\alpha$-Actinin and fascin. J. Biol. Chem. 285:26350-26357. http://dx.doi.org/10.1074/jbc.M110.123117

Dai, J., H.P. Ting-Beall, R.M. Hochmuth, M.P. Sheetz, and M.A. Titus. 1999. Myosin I contributes to the generation of resting cortical tension. Biophys. J. 77:1168-1176. http://dx.doi.org/10.1016/S0006-3495(99)76968-7

Doherty, G.J., and H.T. McMahon. 2008. Mediation, modulation, and consequences of membrane-cytoskeleton interactions. Annu. Rev. Biophys. 37:65-95. http://dx.doi.org/10.1146/annurev.biophys.37.032807.125912

Driscoll, M.K., C. McCann, R. Kopace, T. Homan, J.T. Fourkas, C. Parent, and W. Losert. 2012. Cell shape dynamics: from waves to migration. PLOS Comput. Biol. 8:e1002392. http://dx.doi.org/10.1371/journal.pcbi.1002392

Dunn, G.A., and D. Zicha. 1995. Dynamics of fibroblast spreading. J. Cell Sci. 108:1239-1249.

Erickson, C.A., and J.P. Trinkaus. 1976. Microvilli and blebs as sources of reserve surface membrane during cell spreading. Exp. Cell Res. 99:375384. http://dx.doi.org/10.1016/0014-4827(76)90595-4

Fackler, O.T., and R. Grosse. 2008. Cell motility through plasma membrane blebbing. J. Cell Biol. 181:879-884. http://dx.doi.org/10.1083/jcb.200802081

Farsad, K., and P. De Camilli. 2003. Mechanisms of membrane deformation. Curr. Opin. Cell Biol. 15:372-381. http://dx.doi.org/10.1016/ S0955-0674(03)00073-5

Friedl, P., and K. Wolf. 2009. Proteolytic interstitial cell migration: a fivestep process. Cancer Metastasis Rev. 28:129-135. http://dx.doi.org/ $10.1007 / \mathrm{s} 10555-008-9174-3$ 
Friedl, P., and K. Wolf. 2010. Plasticity of cell migration: a multiscale tuning model. J. Cell Biol. 188:11-19. http://dx.doi.org/10.1083/jcb.200909003

Furcht, L.T., and G. Wendelschafer-Crabb. 1978. Trypsin-induced coordinate alterations in cell shape, cytoskeleton, and intrinsic membrane structure of contact-inhibited cells. Exp. Cell Res. 114:1-14. http://dx.doi.org/ 10.1016/0014-4827(78)90029-0

Gauthier, N.C., M.A. Fardin, P. Roca-Cusachs, and M.P. Sheetz. 2011. Temporary increase in plasma membrane tension coordinates the activation of exocytosis and contraction during cell spreading. Proc. Natl. Acad. Sci. USA. 108:14467-14472. http://dx.doi.org/10.1073/pnas.1105845108

Gilden, J., and M.F. Krummel. 2010. Control of cortical rigidity by the cytoskeleton: emerging roles for septins. Cytoskeleton (Hoboken). 67:477-486.

Golebiewska, U., M. Nyako, W. Woturski, I. Zaitseva, and S. McLaughlin. 2008. Diffusion coefficient of fluorescent phosphatidylinositol 4,5-bisphosphate in the plasma membrane of cells. Mol. Biol. Cell. 19:1663-1669. http://dx.doi.org/10.1091/mbc.E07-12-1208

Hawkins, R.J., R. Poincloux, O. Bénichou, M. Piel, P. Chavrier, and R. Voituriez. 2011. Spontaneous contractility-mediated cortical flow generates cell migration in three-dimensional environments. Biophys. J. 101:1041-1045. http://dx.doi.org/10.1016/j.bpj.2011.07.038

Heit, B., and P. Kubes. 2003. Measuring chemotaxis and chemokinesis: the under-agarose cell migration assay. Sci. STKE. 2003:PL5. http://dx.doi .org/10.1126/stke.2003.170.pl5

Hilgemann, D.W. 2007. Local PIP(2) signals: when, where, and how? Pflugers Arch. 455:55-67. http://dx.doi.org/10.1007/s00424-007-0280-9

Hoffman, B.D., C. Grashoff, and M.A. Schwartz. 2011. Dynamic molecular processes mediate cellular mechanotransduction. Nature. 475:316-323. http://dx.doi.org/10.1038/nature10316

Janmey, P.A., and C.A. McCulloch. 2007. Cell mechanics: integrating cell responses to mechanical stimuli. Annu. Rev. Biomed. Eng. 9:1-34. http:// dx.doi.org/10.1146/annurev.bioeng.9.060906.151927

Kapustina, M., G.E. Weinreb, N. Costigliola, Z. Rajfur, K. Jacobson, and T.C. Elston. 2008. Mechanical and biochemical modeling of cortical oscillations in spreading cells. Biophys. J. 94:4605-4620. http://dx.doi .org/10.1529/biophysj.107.121335

Kasza, K.E., and J.A. Zallen. 2011. Dynamics and regulation of contractile actin-myosin networks in morphogenesis. Curr. Opin. Cell Biol. 23:30-38. http://dx.doi.org/10.1016/j.ceb.2010.10.014

Kasza, K.E., A.C. Rowat, J.Y. Liu, T.E. Angelini, C.P. Brangwynne, G.H. Koenderink, and D.A. Weitz. 2007. The cell as a material. Curr. Opin. Cell Biol. 19:101-107. http://dx.doi.org/10.1016/j.ceb.2006.12.002

Kasza, K.E., F. Nakamura, S. Hu, P. Kollmannsberger, N. Bonakdar, B. Fabry, T.P. Stossel, N. Wang, and D.A. Weitz. 2009. Filamin A is essential for active cell stiffening but not passive stiffening under external force. Biophys. J. 96:4326-4335. http://dx.doi.org/10.1016/j.bpj.2009.02.035

Kruchten, A.E., and M.A. McNiven. 2006. Dynamin as a mover and pincher during cell migration and invasion. J. Cell Sci. 119:1683-1690. http://dx.doi .org/10.1242/jcs.02963

Lipowsky, R. 1991. The conformation of membranes. Nature. 349:475-481. http://dx.doi.org/10.1038/349475a0

Liu, P., T. Sudhaharan, R.M.L. Koh, L.C. Hwang, S. Ahmed, I.N. Maruyama, and T. Wohland. 2007. Investigation of the dimerization of proteins from the epidermal growth factor receptor family by single wavelength fluorescence cross-correlation spectroscopy. Biophys. J. 93:684-698. http:// dx.doi.org/10.1529/biophysj.106.102087

Logan, M.R., and C.A. Mandato. 2006. Regulation of the actin cytoskeleton by PIP2 in cytokinesis. Biol. Cell. 98:377-388. http://dx.doi.org/10 $.1042 / \mathrm{BC} 20050081$

Lorentzen, A., J. Bamber, A. Sadok, I. Elson-Schwab, and C.J. Marshall. 2011. An ezrin-rich, rigid uropod-like structure directs movement of amoeboid blebbing cells. J. Cell Sci. 124:1256-1267. http://dx.doi.org/ $10.1242 /$ jcs. 074849

Machacek, M., L. Hodgson, C. Welch, H. Elliott, O. Pertz, P. Nalbant, A. Abell, G.L. Johnson, K.M. Hahn, and G. Danuser. 2009. Coordination of Rho GTPase activities during cell protrusion. Nature. 461:99-103. http:// dx.doi.org/10.1038/nature08242

Michelot, A., and D.G. Drubin. 2011. Building distinct actin filament networks in a common cytoplasm. Curr. Biol. 21:R560-R569. http://dx.doi .org/10.1016/j.cub.2011.06.019

Mierke, C.T., D. Rösel, B. Fabry, and J. Brábek. 2008. Contractile forces in tumor cell migration. Eur. J. Cell Biol. 87:669-676. http://dx.doi.org/ 10.1016/j.ejcb.2008.01.002

Nelson, R.D., P.G. Quie, and R.L. Simmons. 1975. Chemotaxis under agarose: a new and simple method for measuring chemotaxis and spontaneous migration of human polymorphonuclear leukocytes and monocytes. J. Immunol. 115:1650-1656.
Paluch, E., M. Piel, J. Prost, M. Bornens, and C. Sykes. 2005. Cortical actomyosin breakage triggers shape oscillations in cells and cell fragments. Biophys. J. 89:724-733. http://dx.doi.org/10.1529/biophysj.105.060590

Paňková, K., D. Rösel, M. Novotný, and J. Brábek. 2010. The molecular mechanisms of transition between mesenchymal and amoeboid invasiveness in tumor cells. Cell. Mol. Life Sci. 67:63-71. http://dx.doi .org/10.1007/s00018-009-0132-1

Pesen, D., and J.H. Hoh. 2005. Micromechanical architecture of the endothelial cell cortex. Biophys. J. 88:670-679. http://dx.doi.org/10.1529/ biophysj.104.049965

Pletjushkina, O.J., Z. Rajfur, P. Pomorski, T.N. Oliver, J.M. Vasiliev, and K.A. Jacobson. 2001. Induction of cortical oscillations in spreading cells by depolymerization of microtubules. Cell Motil. Cytoskeleton. 48:235-244. http://dx.doi.org/10.1002/cm.1012

Price, Z.H. 1967. The micromorphology of zeiotic blebs in cultured human epithelial (HEp) cells. Exp. Cell Res. 48:82-92. http://dx.doi.org/10 $.1016 / 0014-4827(67) 90278-9$

Provenzano, P.P., and P.J. Keely. 2011. Mechanical signaling through the cytoskeleton regulates cell proliferation by coordinated focal adhesion and Rho GTPase signaling. J. Cell Sci. 124:1195-1205. http://dx.doi .org/10.1242/jcs.067009

Rangamani, P., M.A. Fardin, Y. Xiong, A. Lipshtat, O. Rossier, M.P. Sheetz, and R. Iyengar. 2011. Signaling network triggers and membrane physical properties control the actin cytoskeleton-driven isotropic phase of cell spreading. Biophys. J. 100:845-857. http://dx.doi.org/ 10.1016/j.bpj.2010.12.3732

Reynolds, E.S. 1963. The use of lead citrate at high $\mathrm{pH}$ as an electron-opaque stain in electron microscopy. J. Cell Biol. 17:208-212. http://dx.doi.org/ 10.1083/jcb.17.1.208

Riedl, J., A.H. Crevenna, K. Kessenbrock, J.H. Yu, D. Neukirchen, M. Bista, F. Bradke, D. Jenne, T.A. Holak, Z. Werb, et al. 2008. Lifeact: a versatile marker to visualize F-actin. Nat. Methods. 5:605-607. http://dx.doi.org/ $10.1038 /$ nmeth. 1220

Rottner, K., and T.E.B. Stradal. 2011. Actin dynamics and turnover in cell motility. Curr. Opin. Cell Biol. 23:569-578. http://dx.doi.org/ 10.1016/j.ceb.2011.07.003

Saarikangas, J., H. Zhao, and P. Lappalainen. 2010. Regulation of the actin cytoskeleton-plasma membrane interplay by phosphoinositides. Physiol. Rev. 90:259-289. http://dx.doi.org/10.1152/physrev.00036.2009

Sahai, E., and C.J. Marshall. 2003. Differing modes of tumour cell invasion have distinct requirements for Rho/ROCK signalling and extracellular proteolysis. Nat. Cell Biol. 5:711-719. http://dx.doi.org/10.1038/ncb1019

Salbreux, G., J.F. Joanny, J. Prost, and P. Pullarkat. 2007. Shape oscillations of non-adhering fibroblast cells. Phys. Biol. 4:268-284. http://dx.doi.org/ 10.1088/1478-3975/4/4/004

Sedzinski, J., M. Biro, A. Oswald, J.Y. Tinevez, G. Salbreux, and E. Paluch. 2011. Polar actomyosin contractility destabilizes the position of the cytokinetic furrow. Nature 476:462-466.

Servant, G., O.D. Weiner, E.R. Neptune, J.W. Sedat, and H.R. Bourne. 1999. Dynamics of a chemoattractant receptor in living neutrophils during chemotaxis. Mol. Biol. Cell. 10:1163-1178.

Sheetz, M.P., J.E. Sable, and H.-G. Döbereiner. 2006. Continuous membranecytoskeleton adhesion requires continuous accommodation to lipid and cytoskeleton dynamics. Annu. Rev. Biophys. Biomol. Struct. 35:417-434. http://dx.doi.org/10.1146/annurev.biophys.35.040405.102017

Steinman, R.M., I.S. Mellman, W.A. Muller, and Z.A. Cohn. 1983. Endocytosis and the recycling of plasma membrane. J. Cell Biol. 96:1-27. http:// dx.doi.org/10.1083/jcb.96.1.1

Strehle, D., J. Schnauss, C. Heussinger, J. Alvarado, M. Bathe, J. Käs, and B. Gentry. 2011. Transiently crosslinked F-actin bundles. Eur. Biophys. $J$. 40:93-101. http://dx.doi.org/10.1007/s00249-010-0621-z

Tseng, Y., E. Fedorov, J.M. McCaffery, S.C. Almo, and D. Wirtz. 2001. Micromechanics and ultrastructure of actin filament networks crosslinked by human fascin: a comparison with $\alpha$-actinin. J. Mol. Biol. 310:351366. http://dx.doi.org/10.1006/jmbi.2001.4716

Tsujioka, M. 2011. Cell migration in multicellular environments. Dev. Growth Differ. 53:528-537. http://dx.doi.org/10.1111/j.1440-169X.2011.01268.x

Vemuri, G.S., J. Zhang, R. Huang, J.H. Keen, and S.E. Rittenhouse. 1996. Thrombin stimulates wortmannin-inhibitable phosphoinositide 3-kinase and membrane blebbing in CHRF-288 cells. Biochem. J. 314:805-810.

Wang, Q., X. Yang, D. Adalsteinsson, T.C. Elston, K. Jacobson, M. Kapustina, and M.G. Forest. 2012. Computational and Modeling Strategies for Cell Motility. In Computational Modeling of Biological Systems: From Molecules to Pathways. N. Dokholyan, editor. Springer, city. 257-296. 